ARTICLE

\title{
Chromosome-level genome assembly of Ophiorrhiza pumila reveals the evolution of camptothecin biosynthesis
}

\author{
Amit Rai (1,2,10凶 ${ }^{\text {, Hideki Hirakawa }}{ }^{3}$, Ryo Nakabayashi ${ }^{4}$, Shinji Kikuchi ${ }^{2,5}$, Koki Hayashi (1) ${ }^{1}$, Megha Rai ${ }^{1}$, \\ Hiroshi Tsugawa (4,6, Taiki Nakaya ${ }^{1}$, Tetsuya Mori ${ }^{4}$, Hideki Nagasaki ${ }^{3}$, Runa Fukushi ${ }^{5}$, Yoko Kusuya ${ }^{7}$, \\ Hiroki Takahashi (10 2,7, Hiroshi Uchiyama ${ }^{8}$, Atsushi Toyoda (1) 9, Shoko Hikosaka ${ }^{2,5}$, Eiji Goto ${ }^{2,5}$, \\ Kazuki Saito (1) ${ }^{1,2,4}$ \& Mami Yamazaki,2四
}

Plant genomes remain highly fragmented and are often characterized by hundreds to thousands of assembly gaps. Here, we report chromosome-level reference and phased genome assembly of Ophiorrhiza pumila, a camptothecin-producing medicinal plant, through an ordered multi-scaffolding and experimental validation approach. With 21 assembly gaps and a contig N50 of $18.49 \mathrm{Mb}$, Ophiorrhiza genome is one of the most complete plant genomes assembled to date. We also report 273 nitrogen-containing metabolites, including diverse monoterpene indole alkaloids (MIAs). A comparative genomics approach identifies strictosidine biogenesis as the origin of MIA evolution. The emergence of strictosidine biosynthesiscatalyzing enzymes precede downstream enzymes' evolution post $\gamma$ whole-genome triplication, which occurred approximately 110 Mya in O. pumila, and before the whole-genome duplication in Camptotheca acuminata identified here. Combining comparative genome anaIysis, multi-omics analysis, and metabolic gene-cluster analysis, we propose a working model for MIA evolution, and a pangenome for MIA biosynthesis, which will help in establishing a sustainable supply of camptothecin.

\footnotetext{
${ }^{1}$ Graduate School of Pharmaceutical Sciences, Chiba University, Chiba, Japan. ${ }^{2}$ Plant Molecular Science Center, Chiba University, Chiba, Japan. ${ }^{3}$ Kazusa DNA Research Institute, Kisarazu, Chiba, Japan. ${ }^{4}$ RIKEN Center for Sustainable Resource Science, Yokohama, Japan. ${ }^{5}$ Graduate School of Horticulture, Chiba University, Chiba, Japan. ${ }^{6}$ RIKEN Center for Integrative Medical Sciences, Yokohama, Japan. ${ }^{7}$ Medical Mycology Research Center, Chiba University, Chiba, Japan. ${ }^{8}$ College of Bioresource Sciences, Nihon University, Fujisawa, Kanagawa, Japan. ${ }^{9}$ Advanced Genomics Center, National Institute of Genetics, Mishima, Shizuoka, Japan. ${ }^{10}$ Present address: RIKEN Center for Sustainable Resource Science, Yokohama, Japan. ${ }^{凶}$ email: amit.rai@chiba-u.jp; 
C ancer is the leading cause of death worldwide, with $70 \%$ of cases occurring in low- and middle-income countries ${ }^{1}$. Among the 30 essential anticancer drugs categorized by the World Health Organization in 2015, several molecules, including topotecan, irinotecan, vincristine, and vinorelbine, are extracted from plants or derived from plant monoterpene indole alkaloids (MIAs), such as camptothecin and catharanthine ${ }^{2,3}$. MIAs are natural products derived from $(S)$-strictosidine, with a monoterpene moiety derived from secologanin, an iridoid class of monoterpenes, and the indole moiety from tryptamine, a decarboxylation product of the amino acid tryptophan (Supplementary Fig. 1). The monoterpenoid moiety of strictosidine then undergoes extensive modifications catalyzed by various enzymes to form diverse MIAs, which represent over 2500 known metabolites $^{2}$. Most of our current understanding of MIA biosynthesis is restricted to the vinca alkaloid synthesis pathway elucidated in Catharanthus roseus ${ }^{4-9}$. Camptothecin, another strictosidinederived molecule and one of the most potent anticancer MIAs, is the precursor for the commercial synthesis of topotecan and irinotecan, and several other camptothecin derivatives are in clinical trials at different stages ${ }^{10,11}$. The camptothecin biosynthesis pathway and the mechanisms regulating its production remain unknown, even though it is one of the most promising plantderived antitumor drugs (Supplementary Fig. 1) ${ }^{11}$. Difficulties in extraction, the low content per gram dry weight of the producing plant tissues, and lack of sustainable resources have limited the development of camptothecin-derived and other anticancer MIAs, contributing to the unaffordability of cancer treatment for most patients.

Ophiorrhiza pumila, a fast-growing herbaceous plant from the Rubiaceae family, has emerged as a model plant for the study of MIA biosynthesis and regulation, and a sustainable source of camptothecin $^{12-14}$. O. pumila hairy roots have been shown to accumulate high levels of camptothecin, and this plant has served as an experimental model for the understanding of MIA biosynthesis for over a decade $3,15,16$. Previous studies have found a correlation of camptothecin biosynthesis and accumulation with the conserved mutation of two amino acids in DNA topoisomerase I in camptothecin-producing plants, including species from the Ophiorrhiza genus, that allow the plants to survive camptothecin cytotoxicity 14,17 . While the basis of natural selection for plant species with resistance against camptothecin is relatively straightforward, how nature simultaneously evolved all the enzymes needed for camptothecin biosynthesis is not yet clear. Understanding MIAs' evolution and biosynthesis is also essential for building sustainable alternate production platforms to facilitate access to these lifesaving compounds. With an estimated 20 million new cancer cases globally by 2025 and an economic burden estimated at $\$ 1.16$ trillion in $2010^{18}$, meeting the increasing demands for camptothecin and other anticancer MIAs has become a daunting challenge and requires immediate attention. A high-quality reference genome for an anticancer MIA-producing plant is the first step toward achieving this goal.

In this study, we show the advantage of ordered multitiered scaffolding with assembly validation at each stage to achieve a highly contiguous genome assembly. This strategy allows us to derive a near-finished and experimentally validated reference and phased genome assembly of $O$. pumila. Our results show the relevance of experimental validation for next-generation plant genome assemblies. Further, we expand the nitrogen-containing metabolome space of Ophiorrhiza by using complete stable isotope labeling and cheminformatics approaches. A combination of comparative genomics approaches suggest the emergence of strictosidine synthase (STR) as a key event in the evolution of strictosidine-derived MIA biosynthesis in plants. Our results suggest that the enzymes involved in the committed step of a specialized metabolite biosynthesis pathway directs evolution and innovation in the plant kingdom. This study, by establishing a high-quality genome and metabolome resource for O. pumila, provides a foundation for yield improvement of valuable anticancer metabolites through synthetic biology and biotechnology.

\section{Results}

Multitiered scaffolding strategy to derive a high-quality plant genome assembly. Assembling a high-quality reference plant genome is challenging due to the inherent heterozygosity, polyploidy, and high repeat content of plant genomes. With the reduced cost of long-read sequencing and advances in scaffolding technologies; however, it has become feasible to achieve plant genome assemblies at the pseudomolecule level ${ }^{19}$. Next-generation sequencing (NGS) technologies, such as Bionano optical maps and Hi-C library sequencing provide valuable orthogonal evidence to validate and improve reference genomes, and to derive chromosome-level genome assemblies ${ }^{20,21}$. Despite this progress, nearly all plant genomes remain highly fragmented, with hundreds to thousands of remaining assembly gaps (Supplementary Fig. 2). In this study, we used a stepwise integration and assembly validation approach using four complementary NGS technologies to derive $O$. pumila de novo genome assembly: PacBio singlemolecule reads $(\sim 122 \times)$, Illumina paired-end reads $(\sim 96 \times)$, Bionano optical mapping $(\sim 250 \times)$, and Hi-C library sequencing ( $90 \times$; Fig. 1a and Supplementary Data 1). A contig-level genome assembly, optimized for different parameters and raw-read lengths using PacBio reads in the Canu assembler ${ }^{22}$, spanned the entire genome in 243 contigs (Supplementary Data 2). Validation of the contig-level assembly using Bionano optical maps identified 15 assembly conflicts (Supplementary Fig. 3), which were manually examined and subsequently corrected. The contig-level genome assembly was subjected to scaffolding using either Bionano or Hi-C libraries, or sequential scaffolding using all possible combinations, i.e., scaffolding first through Bionano and subsequently through $\mathrm{Hi}-\mathrm{C}$, or vice versa. We observed an advantage of sequential scaffolding over a single scaffolding approach, with scaffolding first through Bionano and then by $\mathrm{Hi}-\mathrm{C}$ as the best combination (Table 1). The final Ophiorrhiza genome assembly of $439.90 \mathrm{Mb}$ was achieved within 31 contigs, with contig and scaffold N50 values as 18.49 and $40.06 \mathrm{Mb}$, respectively (Fig. 1a, Table 1, and Supplementary Fig. 4). Full-length chromosome arms were assembled for half of the Ophiorrhiza chromosomes, with only 21 remaining assembly gaps in the entire genome assembly, including the 11 difficult-to-assemble and highly repetitive centromeres (Fig. 1b). We also adopted our sequential scaffolding strategy to achieve phased diploid assembly of Ophiorrhiza using preliminary contig-level assembly derived from Falcon-unzip ${ }^{23}$, resulting in 11 chromosomes with scaffold N50 for haplotig1 and haplotig2 as 40.36 and $42.83 \mathrm{Mb}$, respectively (Supplementary Figs. 4 and 5, and Supplementary Data 3 and 4).

Compared to previously published genome assemblies of anticancer MIA-producing plant species, namely, C. roseus v 2.0 (contig N50: $0.076 \mathrm{Mb})^{6}$, Rhazya stricta (contig N50: $\left.0.08 \mathrm{Mb}\right)^{24}$, Gelsemium sempervirens v 3.0 (contig N50: $0.051 \mathrm{Mb})^{6}$, and Camptotheca acuminata (contig $\mathrm{N} 50: 0.1 \mathrm{Mb})^{25}$, we achieved an improvement of over 180 times from the next best assembly in terms of contig N50 value (Supplementary Fig. 2). Assembly validation, starting from PacBio, followed by Bionano, and finally $\mathrm{Hi}-\mathrm{C}$ provided a stepwise improvement in assembly contiguity. With few remaining genome gaps and contig-level completeness, the Ophiorrhiza reference and phased genomes are, to the best of our knowledge, the most contiguous and complete de novo reference plant genomes to date (Supplementary Fig. 2 and Supplementary Data 5). 
a

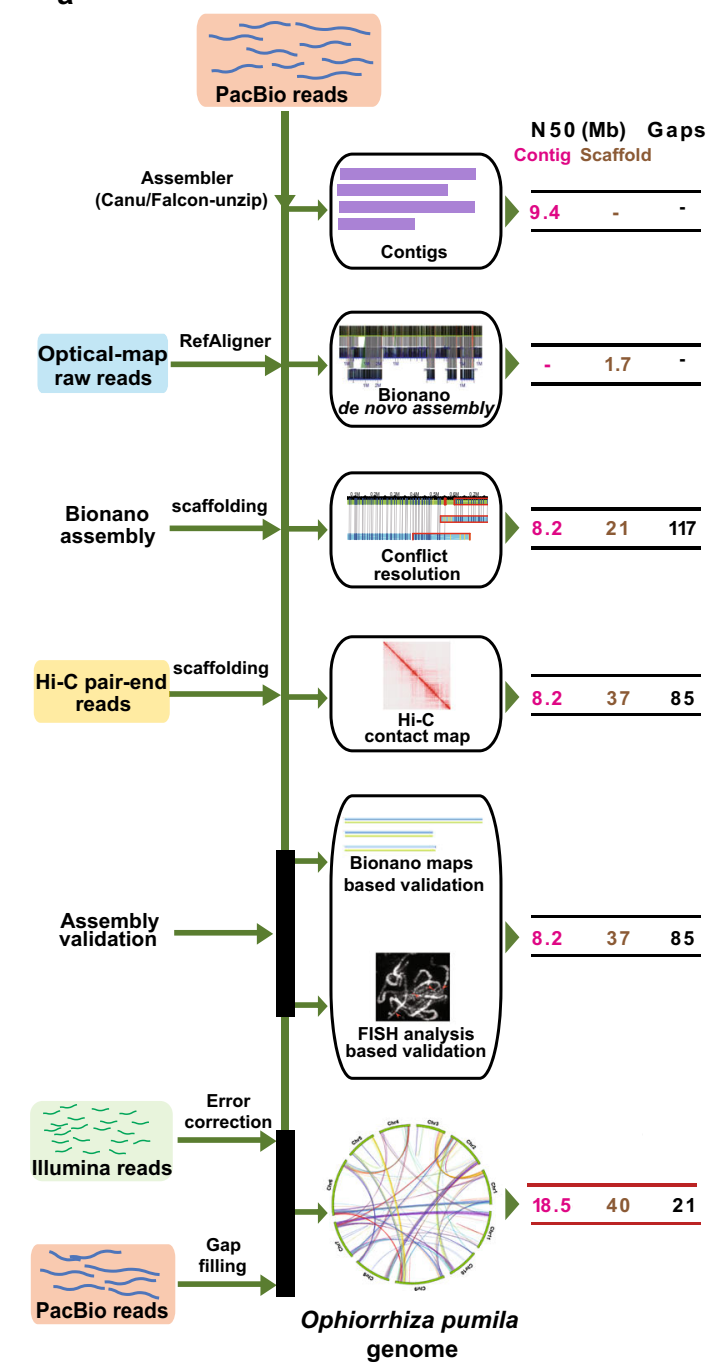

b

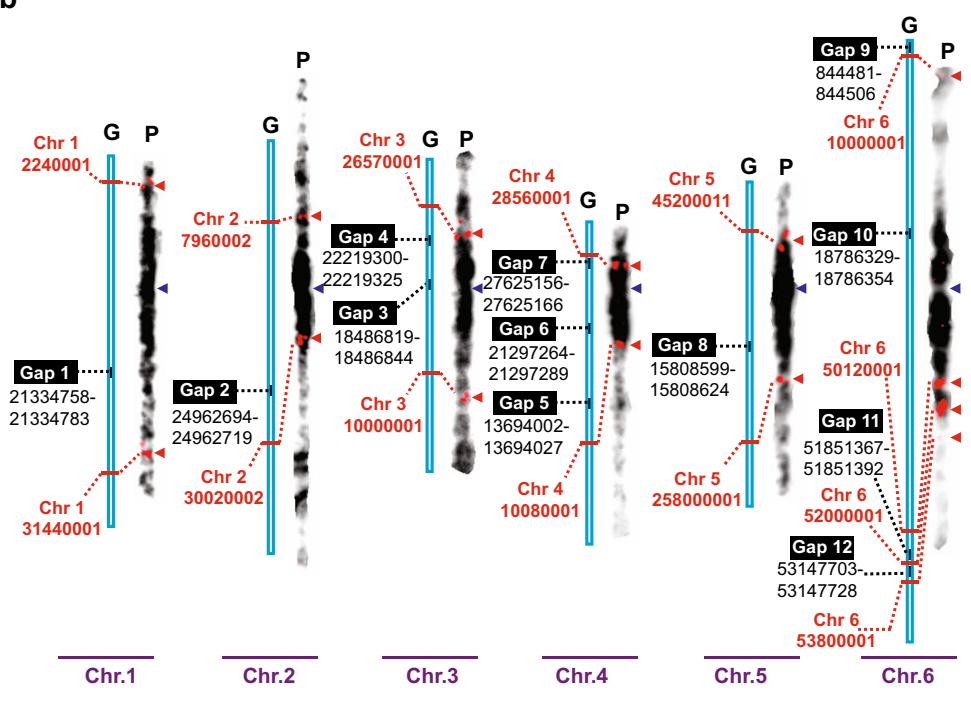

8

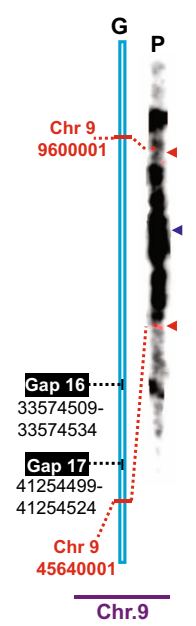

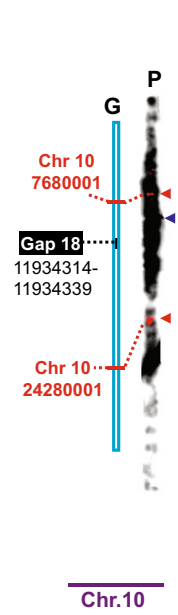

Chr 11 4600001 G P 5702713 Gap 19, 5918930 5918955 Chr $11^{2}$ 6800001 Gap 21 Gap 21 12531525 1550 chr 11 Chr 11
33400001 33400001 . .

\section{.}




\section{Table 10 . pumila reference genome assembly statistics at different stages and combinations of scaffolding.}

\begin{tabular}{|c|c|c|c|c|c|c|c|}
\hline Assembly & $\begin{array}{l}\text { Number of } \\
\text { contigs }\end{array}$ & Number of scaffolds & $\begin{array}{l}\text { Number of contigs } \\
\text { assigned to } \\
\text { scaffolds }\end{array}$ & $\begin{array}{l}\text { Contig } \\
\text { N50 (Mb) }\end{array}$ & $\begin{array}{l}\text { Scaffold } \\
\text { N50 (Mb) }\end{array}$ & $\begin{array}{l}\text { Number } \\
\text { of gaps }\end{array}$ & $\begin{array}{l}\text { Assembly } \\
\text { size (Mb) }\end{array}$ \\
\hline PacBio $^{a}$ only (Canu assembly) & 243 & - & - & 9.38 & - & - & 449.00 \\
\hline Bionano de novo Optical Map & - & 458 & - & - & 1.68 & - & 442.00 \\
\hline PacBio + Opticalb Map & 108 & 45 & 83 & 8.21 & 21.05 & 117 & 442.00 \\
\hline $\mathrm{PacBio}+\mathrm{Hi}-\mathrm{C}^{\mathrm{c}}$ & 213 & 34 & 198 & 9.39 & 40.80 & 96 & 441.00 \\
\hline PacBio + Hi-C + Optical Map ${ }^{d}$ & 239 & 26 & 208 & 8.21 & 24.17 & 91 & 441.90 \\
\hline PacBio + Optical Map $+\mathrm{Hi}-\mathrm{CC}^{\mathrm{e}}$ & 108 & 13 & 108 & 8.21 & 37.11 & 85 & 439.00 \\
\hline $\begin{array}{l}\text { PacBio + Optical Map }+\mathrm{Hi}-\mathrm{C}+\text { PbJelly } \\
\text { (PacBio) + genome polishing (final } O \text {. } \\
\text { pumila reference genome) }\end{array}$ & 31 & $\begin{array}{l}13 \text { (11 Chromosomes + } \\
1 \mathrm{MT}+1 \mathrm{CP})\end{array}$ & 31 & 18.49 & 40.06 & 21 & 439.90 \\
\hline
\end{tabular}

O. pumila is a medicinal plant that can produce the anticancer monoterpene indole alkaloid (MIA) camptothecin. Here, the authors report its genome assembly, and propose a working model for MIA evolution and biosynthesis through comparative genomics, synteny, and metabolic gene cluster analyses.

apacBio refers to contig assembly derived using Pacbio reads only and Canu 22 assembler.

bPacbio + Optical Map refers to Pacbio contig-level assembly scaffolded by Bionano de novo assembly.

${ }^{\mathrm{cPacBio}}+\mathrm{Hi}-\mathrm{C}$ refers to Pacbio contig-level assembly scaffolded by $\mathrm{Hi}-\mathrm{C}$ library sequencing datasets.

dPacBio $+\mathrm{Hi}-\mathrm{C}+$ Optical Map refers to Pacbio $+\mathrm{Hi}-\mathrm{C}$ assembly scaffolded by Bionano de novo assembly.

ePacBio + Optical Map + Hi-C refers to Pacbio + Optical Map assembly scaffolded by $\mathrm{Hi}-\mathrm{C}$ library sequencing datasets.

a
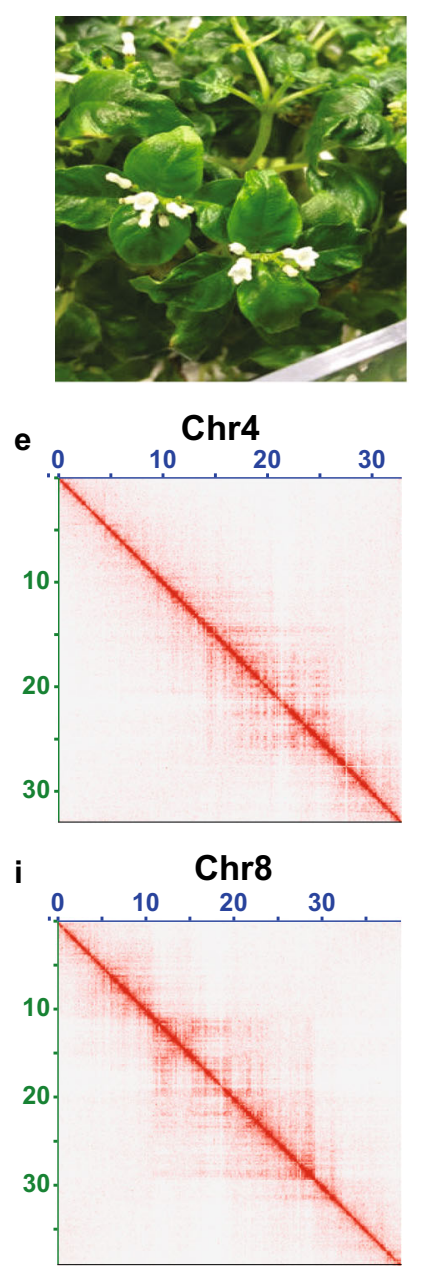

b
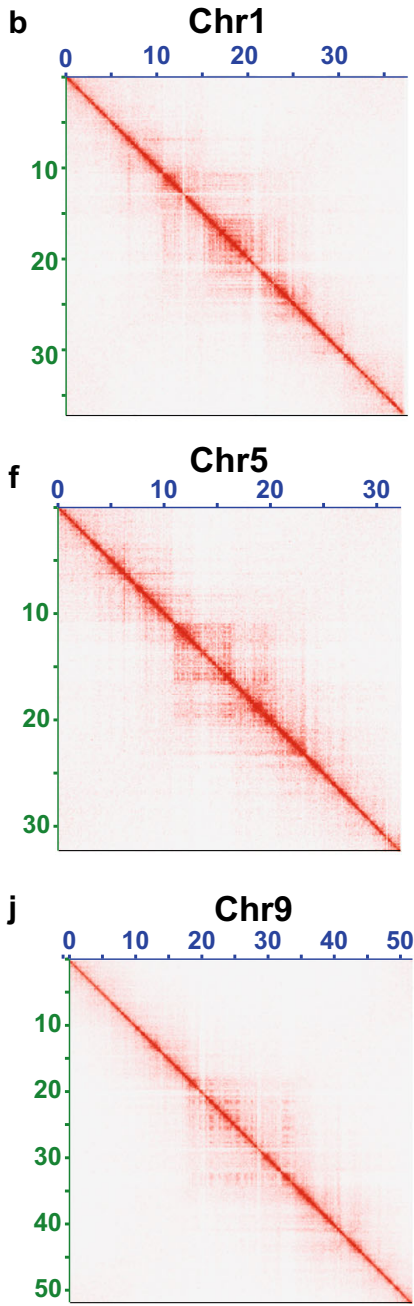

c

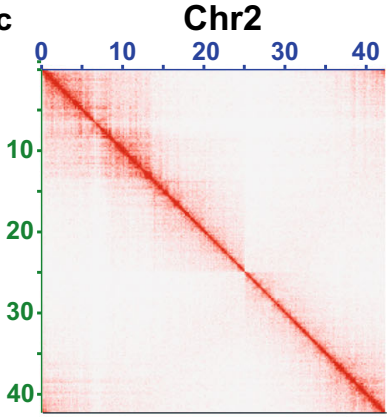

Chr6

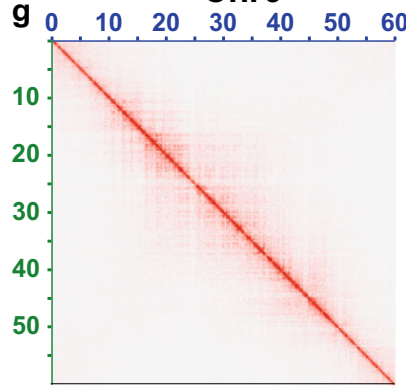

$\mathbf{k}$

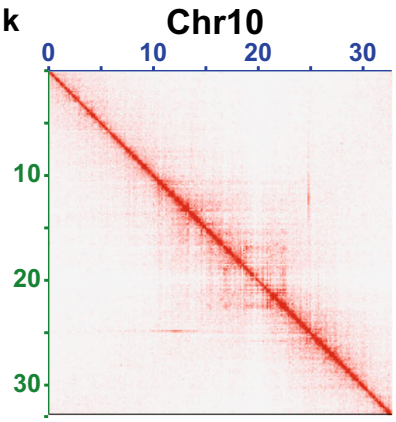

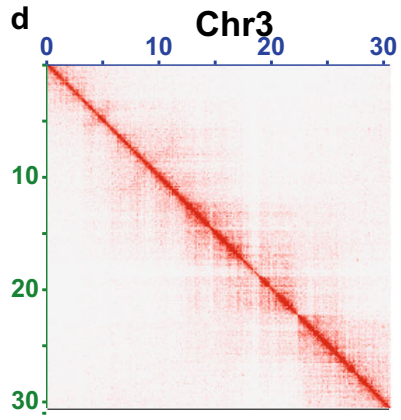

Chr7
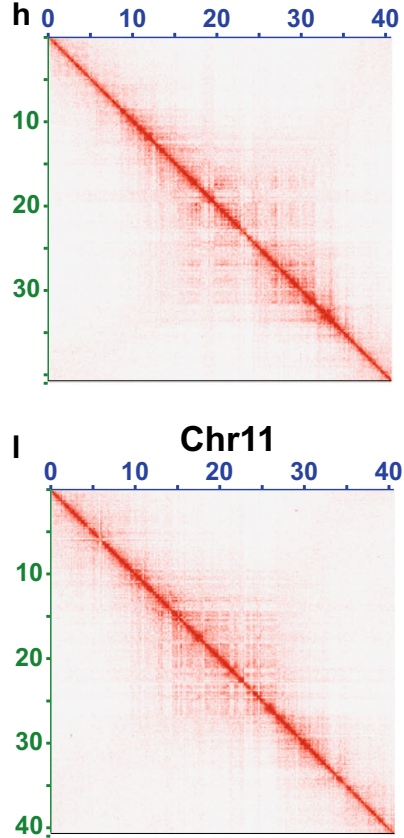

Fig. 2 Hi-C contact map of high-quality chromosome-scale genome assembly of $\mathbf{0}$. pumila. a O. pumila plant under controlled growth conditions. $\mathbf{b}-\mathbf{I} \mathrm{A}$ Hi-

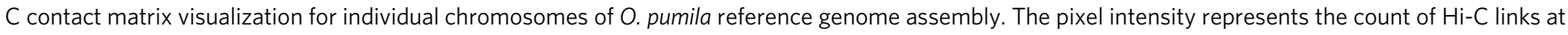
$150-\mathrm{Kb}$ size windows on the chromosomes on a logarithmic scale. Darker red color indicates higher contact probability, while white space represents no or fewer contacts. 
the Ophiorrhiza genome with AEK and $V$. vinifera with minimal rearrangements, with 1 chromosome fission and 11 chromosome fusions, resulting in the present-day karyotype of the Ophiorrhiza genome. Comparison with AEK further supported the accuracy of the karyotypic order of the Ophiorrhiza genome and identified the whole-genome triplication $(\gamma)$ shared among eudicots, with no sign of further genome duplications (Supplementary Fig. 10a-c).

We next compared the Ophiorrhiza genome with the genome of Coffea canephora ${ }^{29}$, also from the Rubiaceae family, which has previously been used as a representative asterid genome for paleogenomic interpretations ${ }^{28}$. Synteny analysis between the Ophiorrhiza and C. canephora genomes showed a potential karyotype rearrangement, with chromosomes 2 and 9 of Ophiorrhiza showing syntenic relationships with chromosome 2 of the coffee genome (Fig. 3a and Supplementary Fig. 10e). The chromosome 2 segment of the coffee genome with a syntenic relationship with chromosome 9 of the Ophiorrhiza genome showed associations with chromosome 13 and chromosome 16 of $V$. vinifera (Fig. 3a). Given that the genome of Ophiorrhiza is near-complete with only 21 assembly gaps, whereas the coffee genome is only $80 \%$ complete and has 7250 assembly gaps, the observed syntenic and potential karyotype rearrangement could very well be a possible misassembly of the coffee genome (Supplementary Fig. 12). Furthermore, the chromosome sizes estimated through FISH analysis supported the Ophiorrhiza genome assembly (Supplementary Fig. 7 and Supplementary Table 1). Therefore, we tested the hypothesis of coffee genome misassembly through FISH analysis in Coffea arabica, an allotetraploid genome resulting from hybridization between $C$. canephora and Coffea eugenioides. FISH analysis using probes designed for the two segments on chromosome 2 of the coffee genome that were in apparent synteny with two chromosomes of the Ophiorrhiza genome produced signals on two different chromosomes instead of on the same chromosome. The FISH analysis suggested a possible misassembly in the coffee genome (Supplementary Fig. 13). Plant genome paleohistorical scenarios and gene-cluster-based specialized metabolite analysis assume accurate gene order for the inputted plant genomes. However, this is most likely not the case for most plant genomes, as very few genomes have been verified. Ophiorrhiza genome assembly provides a valuable resource to validate and improve other plant genomes, and will serve as a model to understand the evolution of genome structure in asterids.

Contrasting genomic features indicate convergent evolution of MIA biosynthesis. The $O$. pumila genome comprises 32,389 gene models, including 778 transcription factors (TFs), 2827 noncoding RNAs, 87 microRNAs (miRNAs), and 493 tRNAs (Fig. 3b, Supplementary Figs. 14 and 15, and Supplementary Data 7-10). The distribution of predicted gene models along the respective chromosomes was in a $\mathrm{V}$-shaped valley form, with low gene density near the centromere for all 11 chromosomes, including for the chromosome 2 after correction based on FISH evidence (Fig. 3b). We used repeat analysis to locate the pericentromeric regions, which were confirmed using FISH analysis (Supplementary Fig. 16). The near-complete genome assembly of Ophiorrhiza also allowed us to identify telomere regions for all eleven chromosomes (Supplementary Data 11). Benchmarking Universal Single-Copy Ortholog (BUSCO) ${ }^{30}$ analysis using the Ophiorrhiza reference and phased genome assemblies showed 97.1\% and $91.2-91.4 \%$ completeness, respectively (Fig. 3c).

Repeat analysis showed 58.17\% Ophiorrhiza genome comprised of transposable elements (TEs), the majority being long terminal repeat (LTR) retroelements, $\sim 75 \%$ of which were classified as
Gypsy-LTRs (Supplementary Data 12). A comparison of TEs across other plant species showed Gypsy-LTR as the dominant repeat class in O. pumila, $R$. stricta, C. roseus, C. canephora, Nicotiana benthamiana ${ }^{31}$, and Solanum lycopersicum ${ }^{32}$, while the C. acuminata genome was dominated by Copia-LTR repeats (Fig. 3d, Supplementary Fig. 17, and Supplementary Data 12). OrthoFinder ${ }^{33}$ analysis-based gene family classification for 33 plant genomes representing broader plant lineages, followed by phylogenetic analysis for single-copy genes using PAML MCMCTREE $^{34}$ software estimated divergence times for Ophiorrhiza from coffee, C. roseus, and C. acuminata at $\sim 47, \sim 68$, and $\sim 120$ Mya, respectively (Supplementary Fig. 18). Synonymous substitutions per synonymous site (Ks) for the paralogs of $O$. pumila, C. roseus, C. acuminata, Arabidopsis thaliana, and coffee genome showed a distinct peak at $\mathrm{Ks}=2$, which represents wellreported and conserved whole-genome triplication across core eudicots $^{35}$ (Fig. 3e and Supplementary Fig. 19). The Ks plot for the paralogs of O. pumila did not show signs of any recent wholegenome duplication (WGD), while the Ks plot for the paralogs of C. acuminata suggested a previously unreported WGD, occurring after the $\gamma$ event, at Ks-peak 0.469, which we estimated at $\sim 42.27$ Mya (Fig. 3e and Supplementary Figs. 18-20). Using Ks values for synteny blocks and orthologs between coffee and Ophiorrhiza, and the estimated divergence time, we determined the Ks per year $(r)$ to be $6.54 \mathrm{e}-9$ for the Rubiaceae.

MIA biosynthesis is known to be remarkably restricted to Gentianales, such as in Rubiaceae ${ }^{24}$. The exceptions are MIA quinolone derivatives, e.g., camptothecin, which is synthesized by Rubiaceae members, such as Ophiorrhiza, as well as by $C$. acuminata in the Cornales. WGDs and TE are regarded as key mechanisms for evolving novel features in plants ${ }^{36-39}$. The differential repeat profiles across the O. pumila, C. roseus, $R$. stricta, and C. acuminata genomes and WGD in C. acuminata suggest different trajectories of acting evolutionary forces, yet resulting in similar chemotypes across MIA-producing plants from the Gentianales and Cornales orders. These results raise the possibility of either a convergent evolution of MIA biosynthesis in otherwise distant plant species or an ancient origin of MIA biosynthesis, which is subsequently lost repeatedly across plant species, while retained by the producing plants.

Diverse indole alkaloids consistent with enzyme families evolved in the Ophiorrhiza genome. Stable isotope labeling, coupled with high-resolution mass spectrometry, offers a powerful approach to assign atom numbers and chemical information to the detected metabolites. It increases the confidence in molecular formula determination for identified metabolite features by eliminating false positives, while considering elemental compositions ${ }^{7,40,41}$. To expand the known chemodiversity of $O$. pumila, particularly nitrogen-containing specialized metabolites, we used a complete ${ }^{15} \mathrm{~N}$-based stable isotope labeling and metabolome analysis approach as previously reported for complete ${ }^{13} \mathrm{C}$-based metabolome labeling for O. pumila and 11 other plant species (Supplementary Fig. 21$)^{7,40}$. Complete ${ }^{15} \mathrm{~N}$ labeling of the Ophiorrhiza metabolome and previously acquired ${ }^{13} \mathrm{C}$-labeled metabolome annotation datasets allowed us to chemically assign 273 nitrogencontaining metabolites, mostly annotated as indole alkaloids (IAs), MIAs, and carboline moieties containing metabolites (Fig. 4, Supplementary Fig. 21, and Supplementary Data 13 and 14). The Ophiorrhiza metabolome showed distinct and diverse nitrogencontaining metabolites, including MIAs, when compared with previously analyzed plant metabolomes (Fig. 4) ${ }^{40}$. The MIA biosynthetic pathways are well conserved across producing plant species and are derived from strictosidine (or strictosidinic acid, in the case of C. acuminata $)^{42}$. Along with derivatives of strictosidine, 


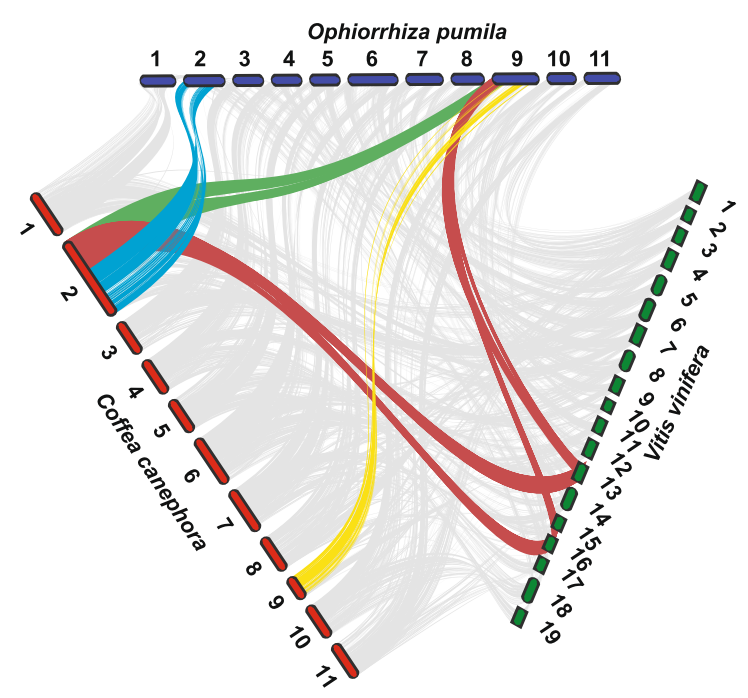

c

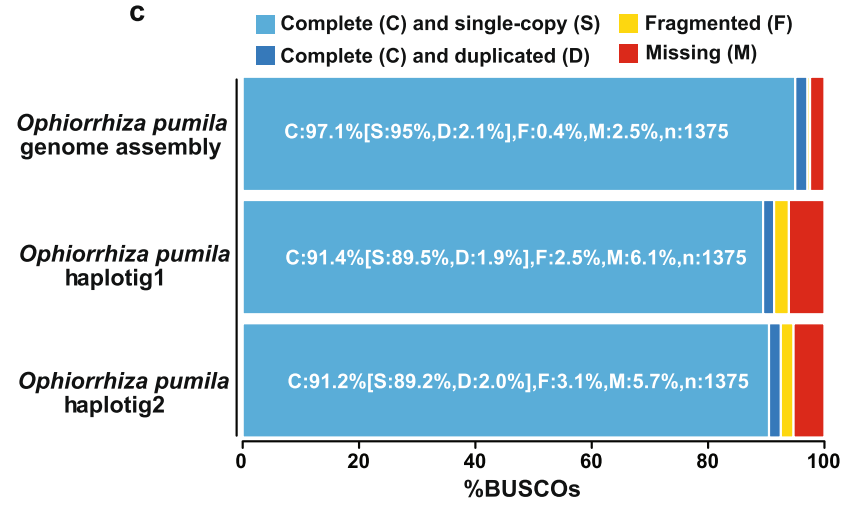

d
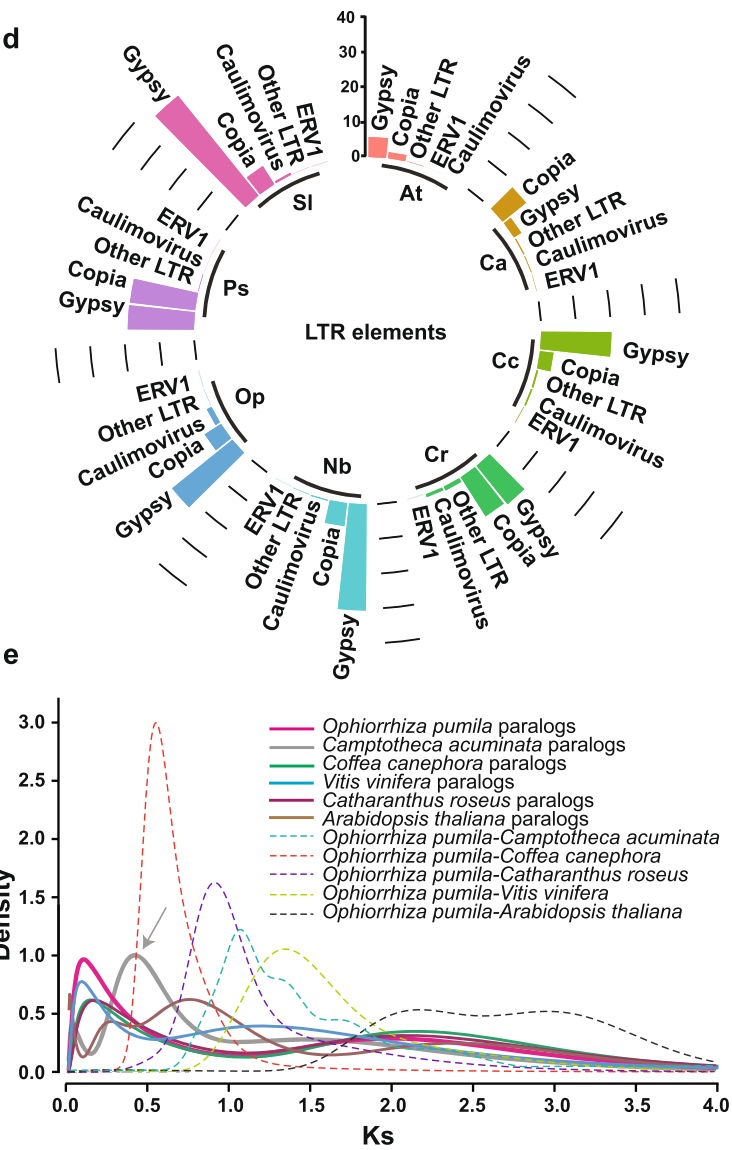

11 b

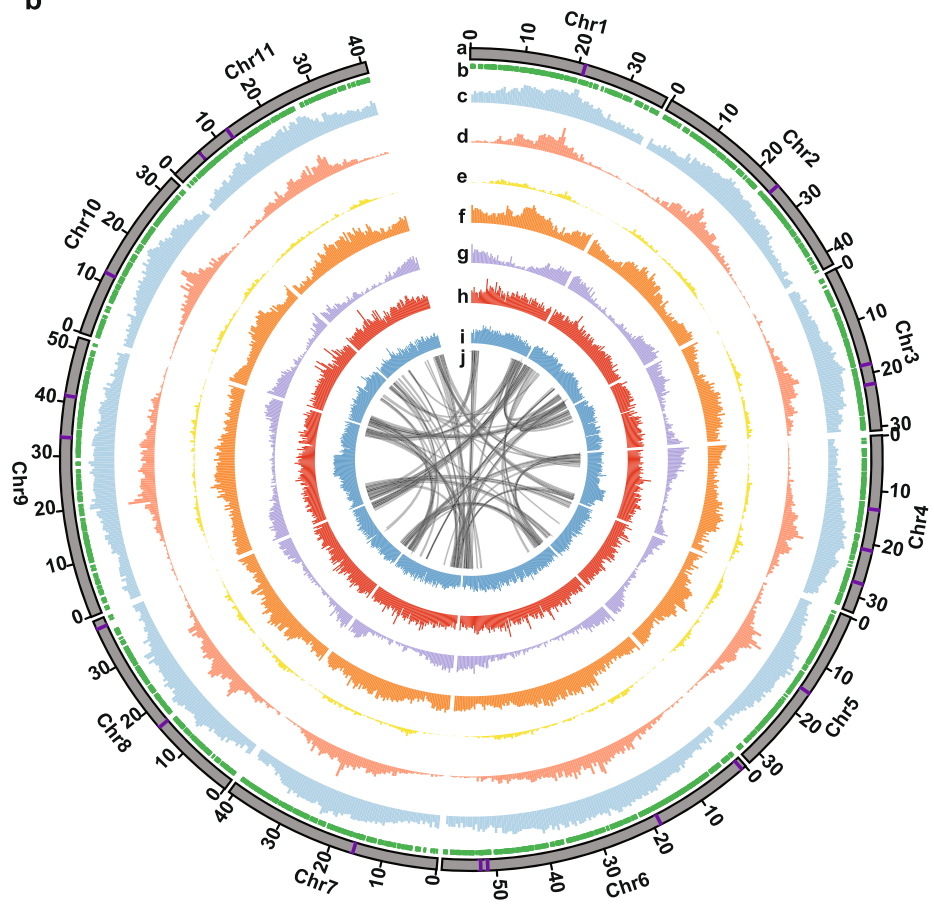

Fig. 3 O. pumila genomic landscape and convergent evolution of monoterpene indole alkaloid biosynthesis. a Synteny blocks between $O$. pumila, $C$. canephora, and V. vinifera. Synteny analysis suggested karyotypic rearrangement between chromosomes 2 and 9 of $O$. pumila and C. canephora genomes, respectively. $\mathbf{b}$ Characteristics of the 11 chromosomes of $O$. pumila. Track a-c corresponds to chromosomes (assembly gap is depicted by purple line at each chromosome), phasing blocks, and repetitive sequences, respectively. Track d-i corresponds to the distribution of long terminal repeat (LTR)-Gypsy, distribution of LTR-Copia, GC density, distribution of predicted gene models, SNP density, and indel density, respectively. Track j corresponds to syntenic blocks. The bar, representing chromosomes, are scaled to chromosome lengths. c Evaluation of $O$. pumila genome assemblies using Benchmarking Universal Single-copy Orthologs (BUSCO) analysis. d Percentage of genome content comprising LTR elements for eight plant species. At Arabidopsis thaliana, Ca Camptotheca acuminata, Cc Coffea canephora, Cr Catharanthus roseus, Nb Nicotiana benthamiana, Op O. pumila, Ps Papaver somniferum, SI Solanum lycopersicum. e Synonymous substitution rate (Ks) distribution plot for paralogs and orthologs of $O$. pumila with other eudicots as shown through colored continuous and dotted lines, respectively. The arrow highlights the recent whole-genome duplication identified in C. acuminata genome. Source data are provided as a Source data file.

camptothecin, and known intermediates of the camptothecin biosynthetic pathway (Supplementary Fig. 1), MIAs such as eburnamonine, nothapodytine, vincamenine, and D-glucopyranosyl vincosamide have also been identified in Ophiorrhiza. Specialized metabolite classes, including MIAs, IAs, and anthraquinones, accumulated in a tissue-specific manner, with the highest levels in the root and hairy root, and low levels in the leaf tissues (Supplementary Figs. 22 and 23, and Supplementary Data 15). Several of the assigned MIAs were also reported previously in C. roseus, G. sempervirens, and C. acuminata ${ }^{7}$. Consistent with MIA accumulation, gene expression analysis showed tissue-specific expression of secoiridoid biosynthesis-related genes, with high expression 


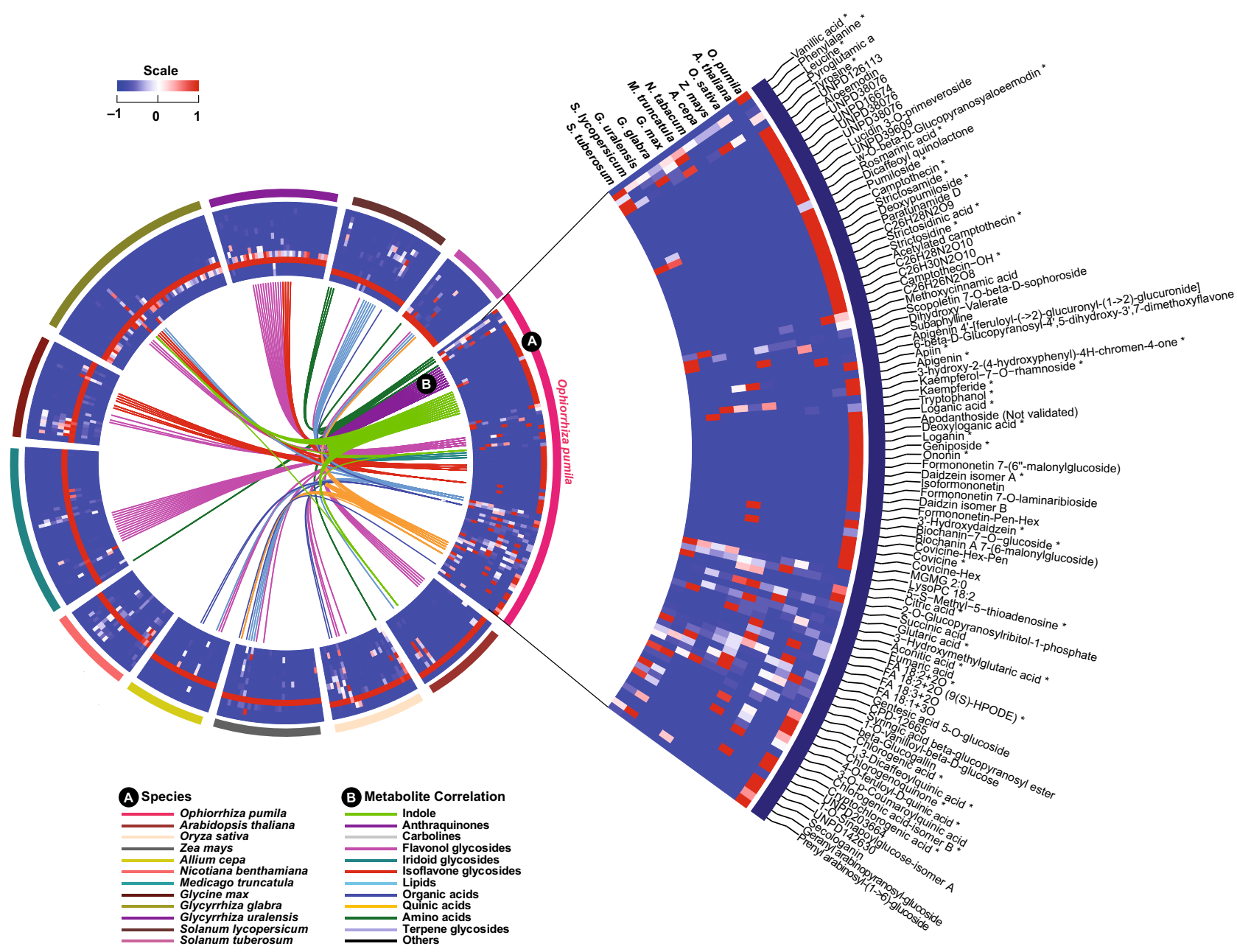

Fig. 4 Ophiorrhiza pumila metabolome, assigned using ${ }^{13} \mathrm{C}$ and ${ }^{15} \mathrm{~N}$ stable isotope labeling, compared with metabo-space of 11 plant species. Circuit $A$ shows plant species used to compare metabolome space with that of $O$. pumila, and circuit B shows the connections between metabolite features, and are based on metabolite network relationships defined by a correlation coefficient $>0.85$. Highly accumulated metabolites across 12 plant species and their relationships in the form of metabo-ontology and scaled accumulation levels as a heat map are shown here. Metabolites were filtered (log10 intensity $>3.9$ ) and assigned to the O. pumila category. If a metabolite was not detected in O. pumila, then it was assigned to the plant category with the highest accumulation compared to the rest of the plant species. *Indicates chemically assigned metabolites based on pure standards or MS/MS analysis using public databases. Source data are provided as a Source data file.

in the root and hairy root, and low expression in the leaf and cell suspension culture of O. pumila (Supplementary Figs. 1, 24 and 26, and Supplementary Data 16-18). Expression analysis showed that homologs of genes associated with secoiridoid and MIA biosynthesis were highly coexpressed, and were considered strong candidate MIA biosynthesis genes in O. pumila. Secoiridoid biosynthesis genes were highly coexpressed with homologs of MIA biosynthesisassociated enzymes, including 10-hydroxycamptothecin $O$ methyltransferase, O-acetylstemmadenine oxidase (ASO/PAS), polyneuridine-aldehyde esterase (PNAE), perakine reductase $(P R)$, rankinidine/humantenine-11-hydroxylase $3(R H 11 H)$, sarpagan bridge enzyme ( $S B E)$, strictosidine beta-D-glucosidase ( $S G D)$, tabersonine-19-hydroxy-O-acetyltransferase (T19AT), tabersonine 3-oxygenase, and tetrahydroalstonine synthase (THAS; Supplementary Figs. 25-27). Integration of metabolome and transcriptome profiling for multiple tissues of Ophiorrhiza identified strong candidate MIA biosynthesis genes (Supplementary Fig. 27 and Supplementary Data 19). The expression of genes associated with secoiridoids and MIA biosynthesis showed a strong correlation with MIA accumulation, indicating that the Ophiorrhiza root and hairy roots are the sites of active biosynthesis (Supplementary Figs. 23, 26 and 27, and Supplementary Data 19).
To understand the features of conserved gene sets associated with MIA biosynthesis and their evolution, we compared the Ophiorrhiza genome with that of 12 plant species, including three MIA-producing plant genomes (C. acuminata, C. roseus, and G. sempervirens). Using OrthoFinder-based gene classification, we identified a total of 15,943 orthogroups shared among the four MIA-producing plants, with $64.8 \%$ of orthogroups being common to all four species and 513 orthogroups being specific to the MIA-producing plants (Supplementary Fig. 28 and Supplementary Data 20). Gene ontology (GO) enrichment analysis of the MIA-specific orthogroups showed dioxygenase (OG:0016701) and oxidoreductase activities (OG:0051213), biochemical reactions essential for MIA biosynthesis and diversification, as the significantly enriched GO terms (Supplementary Fig. 28c). The orthogroups were further analyzed to infer the ancestral and lineage-specific gene content along the phylogenetic tree, and to calculate posterior probabilities for gene family evolution and dynamics. Compared to Ophiorrhiza and other MIA-producing plants, the C. acuminata genome showed massive gene expansion, which is consistent with the WGD identified in this study. Overall, the Ophiorrhiza genome showed gain and expansion for 1047 and 1225 orthogroups, 


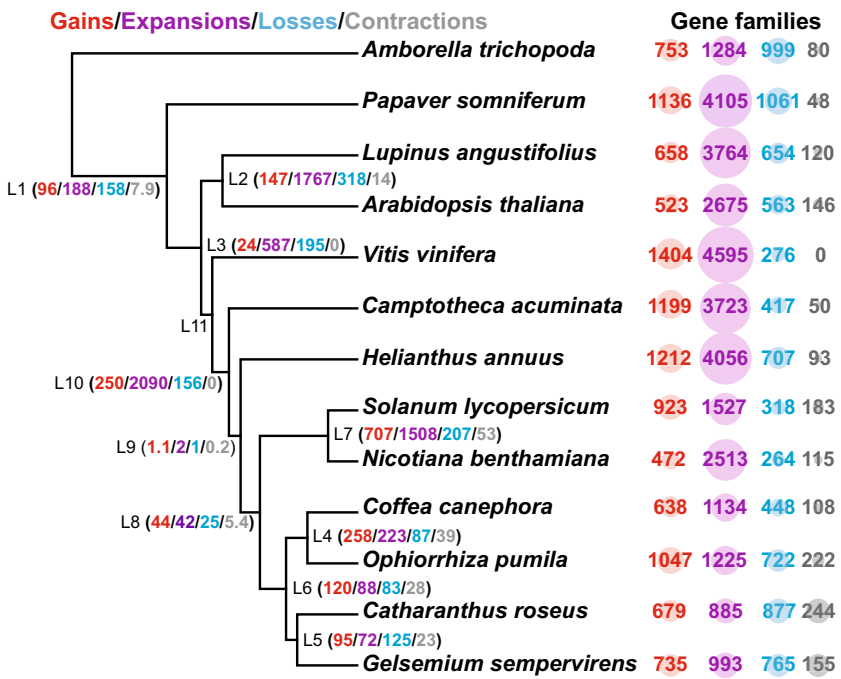

Fig. 5 Evolution as gain (red), expansion (purple), loss (blue), or contraction (gray) of the orthogene families in context of phylogenetic profile. Count software was used to calculate posterior probabilities to capture the dynamics of evolution through the reconstruction of ancestral gene content and changes at the key nodes, which correspond to the lineage-specific gene characteristics. The circle radius is scaled based on the number of genes assigned to a specific category. Source data are provided as a Source data file.

respectively (Fig. 5 and Supplementary Data 21), and GO enrichment analysis showed significant enrichment of processes associated with dioxygenase activity, hydrolase activities, hydroxyl-methyl glutaryl-CoA, and oxidoreductase activities. Several genes assigned to secoiridoid and MIA biosynthesis and highly correlated with metabolite accumulation patterns in Ophiorrhiza were among the gene families expanded or gained specifically to MIA-producing plants (Supplementary Data 1921). Unless there exists a completely independent pathway toward the biosynthesis of MIAs across producing plants, an unlikely scenario, the shared chemotype suggests the possible existence of conserved gene families and secondary metabolite gene clusters within these plant genomes, with positive gene selection or gene expansion occurring during the evolution of MIA biosynthesis $^{2,7,42}$.

Strictosidine biogenesis is the driving force of MIA evolution in plants. Orthogene families with genes assigned to early secoiridoid biosynthesis pathway (Supplementary Fig. 26a), including geraniol synthase (GES), geraniol 10-hydroxylase $(G 10 H)$, 10-hydroxygeraniol oxidoreductase (10-HGO), iridoid synthase, iridoid oxidase, 7-deoxyloganetin glucosyltransferase (7-DLGT), and 7-deoxyloganic acid 7-hydroxylase (7-DLH), were well conserved and uniformly represented in all 13 plant genomes (Fig. 6a). However, gene families corresponding to late secoiridoid pathway genes, including loganic acid $O$-methyltransferase $(L A M T)$ and secologanin synthase (SLS), were specifically gained and expanded in MIA-producing plants. While orthogroups corresponding to LAMT (OG0000252) and SLS (OG0002438) were retained in all species, OG0014621 (LAMT) was specifically gained in O. pumila, and OG0013616 (SLS) was specifically gained and expanded in the $C$. acuminata, $C$. roseus, and Ophiorrhiza genomes (Fig. 6a).

The generation of strictosidine is the committed step in the biosynthesis of MIAs in Gentianales. Orthogene-based classification of genes showed that STR coding enzymes were divided into two distinct groups. Orthogene family OG0000148 was represented in all plants except Papaver somniferum and Amborella trichopoda, while OG0015245, which included all known and functionally characterized genes for strictosidine synthesis, was specifically gained and expanded in Gentianales, including those from $O$. pumila, C. roseus, and G. sempervirens (Fig. 6a and Supplementary Data 21). Compared to $C$. roseus and $G$. sempervirens, which included single-copy genes in the orthogene family OG0015245, the $O$. pumila genome included two genes resulting from tandem duplication, namely, Opuchr05_g0008300 and Opuchr05_ g0008180. Gene family analysis of functional STR homologs of O. pumila across the genomes of 32 plant species showed no representatives from ancient plant genomes, monocots, and AEK (Supplementary Fig. 29). The biosynthesis of MIAs in $C$. acuminata was previously attributed to strictosidinic acid and not strictosidine, which supports the absence of genes in orthogroup OG0015245 42,43

An alternate pathway for MIA biosynthesis in C. acuminata is through strictosidinic acid, which is synthesized by the condensation of secologanic acid with tryptamine ${ }^{42}$. Recently, Yang et al. functionally characterized Cac_g012666.t1 (CYP72A610) and Cac_g017137.t1 (CYP72A565) as bifunctional SLS-like enzymes, catalyzing the synthesis of loganic acid from 7-deoxyloganic acid and subsequently to secologanic acid ${ }^{43}$. The tryptophan decarboxylase (TDC) enzyme, which synthesizes tryptamine from tryptophan, is represented by the orthogene OG0000823. Unlike orthogenes representing functional STR and SLS, OG0000823 was present in all plant species that we analyzed, suggesting an essential role of TDC in amino acid metabolism in plants. Phylogenetic analysis for orthogenes representing SLS and STR showed genes being assigned to two distinct groups, those present specifically in MIA-producing plants and those that are also present in the non-MIA-producing plants (Fig. 6b-d). Similar to functionally characterized STR and SLS enzymes, orthogenes corresponding to enzymes associated with MIA biosynthesis, such as SGD, THAS, SBE, T19AT, PNAE, ASO/ PAS, and PR, were also specifically expanded in Ophiorrhiza and at least one of the other three MIA-producing plant genomes (Fig. 6a and Supplementary Data 21). Phylogenetic analysis of these specialized enzymes also showed MIA plant-specific gene family formation, which included previously functionally characterized enzymes involved in MIA biosynthesis (Supplementary Figs. 30-40). Phylogenetic analysis and gene gain/expansion analysis showed a positive selection of gene sets specific to MIAproducing plants. Moreover, the emergence of specialized enzymes, including STR and bifunctional SLS, specific to MIAproducing plants and catalyzing the synthesis of strictosidine showed the importance of strictosidine biogenesis in facilitating the evolution of novel enzymes for MIA biosynthesis and diversification.

WGDs and small-scale duplications (SSDs) are the major source of evolutionary novelty, providing gene pools to evolve new or specialized functions, and also play an important role in speciation ${ }^{44-46}$. Theoretical models for the evolutionary trajectories of duplicated genes propose that, in most cases, one copy of the duplicated gene retains the original function, while another copy neutrally evolves without any selective constraints, thus resulting in its inactivation due to the accumulation of deleterious mutations or even deletion ${ }^{47}$. In a small fraction of cases, the duplicate gene undergoes gain-of-function mutations and is retained through positive selection forces ${ }^{46}$. The native genes undergo a rapid rate of mutation and thus should have a lower Ks value than the ancestral genes, resulting in the emergence of a new enzyme with a novel function. However, in contrast to this hypothesis, the median Ks for enzymes associated with secoiridoids and MIA biosynthesis in both MIA-producing and nonproducing plants was 1.586 , which corresponds to the Ks 


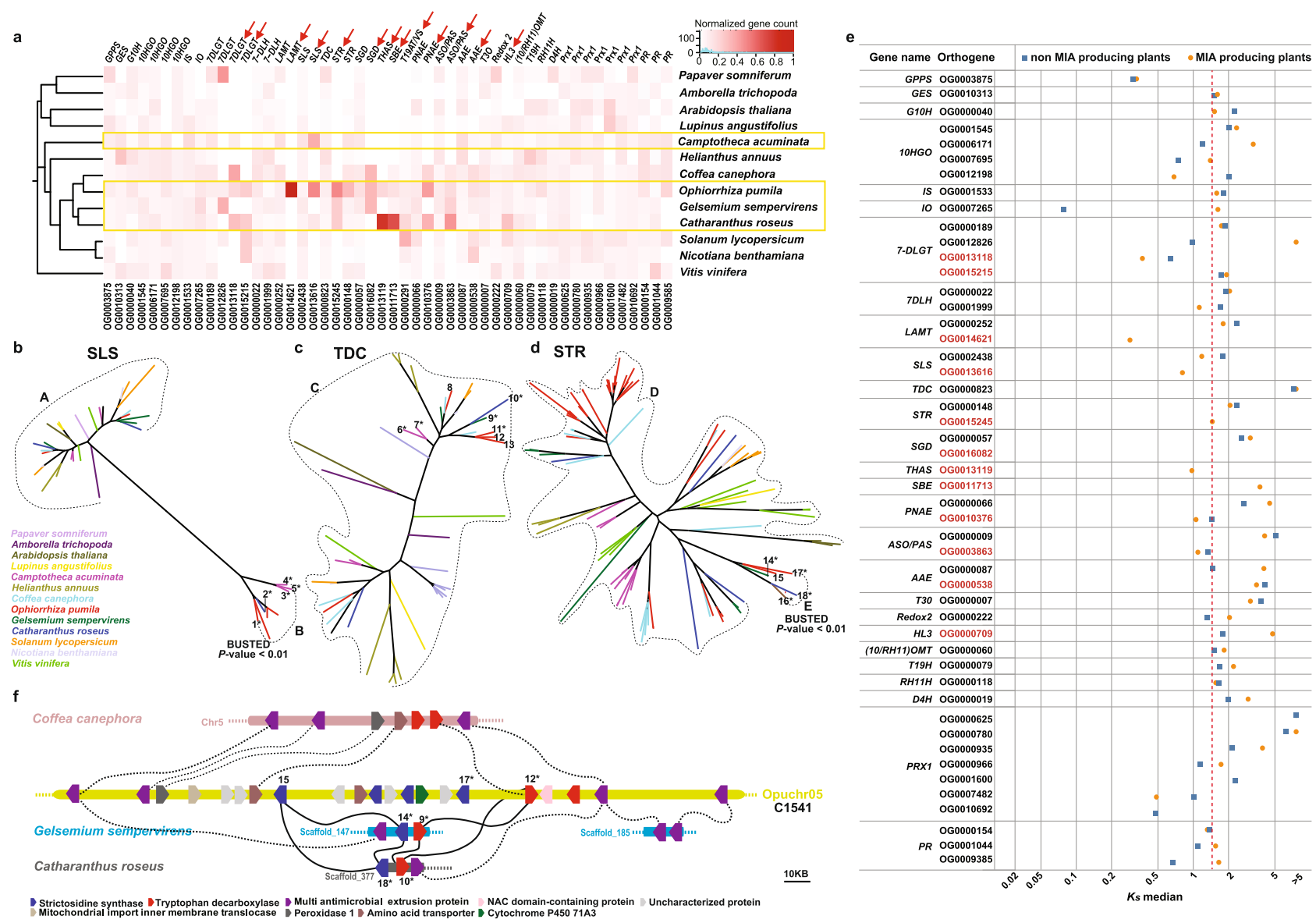

Fig. 6 Emergence of strictosidine synthase, the starting point for the evolution of monoterpene indole alkaloid biosynthesis in plants. a Normalized gene count data for orthogene families assigned to MIA biosynthesis across 13 plant species. The yellow box indicates MIA-producing plants. Normalization was performed by dividing gene count data for a specific plant species with total number of genes assigned to a given orthogene family. The red arrows at the top of gene names highlight the orthogene families specifically gained or expanded in the MIA-producing plant species. b-d Maximum likelihood phylogenetic tree based on genes from orthogene OG0002438(A) and OG0013616(B), representing SLS; orthogene OG0000823(C), representing TDC; and orthogene OG0000148 ${ }^{(D)}$ and OG0015245 ${ }^{(E)}$, representing STR coding genes. BUSTED analysis was performed using genes in the group $B$ as test set and the genes in the group A as the background for SLS, and genes in the group E as test set against genes in the group D as the background for STR. *Functionally characterized genes. 1: Opuchr02_g0013060-1.1; 2: CRO_T109448; 3: Cac_g017137.t1; 4: Cac_g012666.t1; 5: Cac_g012664.t1; 6: Cac_g023139.t1; 7: Cac_g018974.t1; 8: Opuchr05_g0005520-1.1; 9: Gs_TDC; 10: CRO_T125328; 11: Opu_chr01_g0011270-1.1; 12: Opuchr05_g0008370-1.1; 13: Opuchr01_g0009570-1.1; 14: Gs_STR; 15: Opuchr05_g0008180-1.1; 16: AAY81922; 17: Opuchr05_g0008300-1.1; 18: CRO_T125329. e Median synonymous substitutions per synonymous sites (Ks) distribution for genes associated with secoiridoid and MIA biosynthesis pathways across MIA-producing and non-MIA-producing plants. Dotted red line refers to Ks median for functional STR in Ophiorrhiza genome. Orthogenes highlighted by red color are the orthogenes specifically gained or expanded in MIA-producing plants as shown in a. f Conserved gene clusters, C1541, essential for the biosynthesis of strictosidine-derived MIAs. Dashed lines show syntenic blocks, while scaffolds for each genome are shown through distinct color. GPPS geranyl diphosphate synthase, GES geraniol synthase, G10H geraniol 10-hydroxylase, 10-HGO 10-hydroxygeraniol oxidoreductase, IS iridoid synthase, 10 iridoid oxidase, 7-DLGT 7-deoxyloganetin glucosyltransferase, 7-DLH 7-deoxyloganic acid 7-hydroxylase, LAMT loganic acid Omethyltransferase, SLS secologanin synthase, TDC tryptophan decarboxylase, STR strictosidine synthase, SGD strictosidine beta-D-glucosidase, THAS tetrahydroalstonine synthase, PNAE polyneuridine-aldehyde esterase, ASO/PAS O-acetylstemmadenine oxidase, AAE acetylajmaline esterase, TEX1tabersonine 6,7-epoxidase 1, HL3 hydrolase 3, 10OMT 10-hydroxycamptothecin O-methyltransferase, T19H tabersonine-19-hydroxy-Oacetyltransferase, $R H 11 \mathrm{H}$ rankinidine/humantenine-11-hydroxylase 3, D4H deacetoxyvindoline 4-hydroxylase, Prx 1 peroxidase 1, PR perakine reductase. Source data are provided as a Source data file.

median for OG0015245 (STR; Fig. 6e). MIA-producing plants showed a lower Ks median than non-MIA-producing plants for OG000040 (G10H), OG0012198 (10-HGO), OG0013118 (7DLGT), OG0014621 (LAMT), OG0013616 (SLS), OG0015245 (STR), OG0011713 (THAS), OG0010376 (PNAE), OG0003863 (ASO), and OG0007482 (PRX1), suggesting a higher rate of substitution and evolution for these specialized enzymes. The median Ks for TDC, an enzyme essential for tryptophan metabolism across all plant species, showed a similar substitution rate in MIA- and non-MIA-producing plants (Fig. 6e and Supplementary Data 22). For camptothecin-producing plants, the median Ks for genes associated with MIA biosynthesis was significantly smaller in O. pumila and C. acuminata but higher in the coffee genome, which shares otherwise high genome collinearity and sequence similarity with the Ophiorrhiza genome (Supplementary Fig. 41). A higher median Ks for the MIAassociated orthogene families in the coffee genome suggests an ancient origin for the genes that otherwise would have been actively evolving, as suggested by the smaller median Ks value. Phylogenetic analysis by maximum likelihood test and BUSTED analysis ${ }^{48}$ for orthogene families gained or expanded in MIAproducing plant genomes indicated positive selection for SLS 
(OG0013616) and STR (OG0015245) specific to the MIAproducing plant species (Fig. 6b, d).

Evolution of MIA biosynthesis is centered around secondary metabolite gene clusters. To gain insight into whether the physical location plays a role in positive selection and expansion of genes associated with MIA biosynthesis, we performed gene cluster analysis in the Ophiorrhiza genome. In total, we identified 358 metabolic gene clusters in the O. pumila genome, representing 3551 gene models across 11 chromosomes (Supplementary Data 23). Coexpression analysis for a given gene cluster showed a low Pearson correlation coefficient (PCC) value, with the median PCC value for $3 / 4^{\text {th }}$ of the gene clusters being $<0.3$ (Supplementary Data 24). We identified metabolic gene clusters, such as C1394, C1620, C1708, C1709, C1909, C1925, and C1959, which included 7-18 gene members, and were highly coexpressed. Using the presence of at least one orthogene family associated with MIA biosynthesis pathways from O. pumila gene models in the identified gene clusters as the selection criteria, we assigned 33 gene clusters as putative MIA gene clusters (Supplementary Data 18, 23 and 24). While MIA biosynthesisassociated genes were highly coexpressed, MIA gene clusters showed low coexpression values among member genes. Among the MIA gene clusters, $\mathrm{C} 1385$ and $\mathrm{C} 1749$ showed coexpression between member genes. Although our results showed low coexpression coefficients for genes within a gene cluster, we observed 261 gene clusters with at least one pair of genes having PCC values over 0.7 . The fact that several of these gene clusters included genes with no expression within the tissues analyzed was one of the reasons for the low PCC scores within individual gene clusters. The behavior of the identified gene clusters and associated coexpression values were similar to previously reported trends in other plant genomes ${ }^{49,50}$, suggesting a lack of widespread coexpression among member genes of associated gene clusters in plants.

Out of 358 secondary metabolite gene clusters identified, 91 gene clusters included at least one orthogroup specific to MIAproducing plants (Supplementary Data 20 and 23-26). One of the key gene clusters identified in the Ophiorrhiza genome was C1541, which included functionally characterized TDC (Opuchr05_g0008370-1.1) and STR (Opuchr05_g0008300-1.1 and Opuchr05_g0008180-1.1) together with enzymes annotated as amino acid transporters, cytochrome P450 71A3, NAC domain-containing protein, and multi-antimicrobial extrusion (MATE) protein (Fig. 6f). Previously, C. roseus and G. sempervirens genome analysis have also reported the presence of a STR-TDC-MATE gene cluster ${ }^{6}$. Synteny analysis showed conserved synteny between the C. roseus and G. sempervirens genomes at $\mathrm{C} 1541$, which included both functionally characterized STR, TDC, and MATE proteins. The coffee genome, which shares significant collinearity and sequence similarity with the Ophiorrhiza genome, also showed conserved gene cluster collinearity with C1541 (Fig. 6f, and Supplementary Figs. 12 and 15). However, the relatively conserved functional STR orthogenes present across strictosidine-derived MIA-producing plants at gene cluster C1541 were lost in the coffee genome, while other features of the gene cluster, including TDC coding enzymes, were retained (Fig. 6f). Comparative genome analysis between Ophiorrhiza and coffee showed high sequence similarity and gene localization along chromosomes, yet loss of functional STR within the coffee genome at the gene cluster may have limited the opportunity to direct evolution toward MIA biosynthesis, which also explains the higher median Ks for enzymes associated with MIA biosynthesis (Supplementary Fig. 41). The entire secoiridoid biosynthetic pathway and MIA biosynthesis-associated genes from the Ophiorrhiza genome were present in 29 out of 33 MIA gene clusters (Supplementary Fig. 26). The association of coexpressed genes assigned to secoiridoid and MIA biosynthesis pathways with gene clusters was statistically significant based on two-sided Fisher's exact test with corrected $p$ value $<0.05$. Furthermore, at least one-third of the member genes of 20 out of the 33 MIA gene clusters showed conserved collinearity in the C. roseus, C. acuminata, and G. sempervirens genomes (Fig. 7, Supplementary Figs. 42-48, and Supplementary Data 26). The gene cluster C1693 exhibited conserved synteny for functionally characterized genes encoding 10-HGO, ASO/PAS, and THAS3 in C. roseus, while an adjacent gene cluster, C1684, showed conserved synteny for the functionally characterized $G 10 \mathrm{H}$ in O. pumila and C. roseus (Supplementary Fig. 46). Synteny between $O$. pumila and C. roseus or G. sempervirens genomes centered around gene clusters was statistically significant based on Fisher's exact test ( $p$ value $<0.05$ ), suggesting gene clusters as the critical genomic regions for the evolution and expansion of specialized metabolites (Supplementary Data 26). The median Ks for gene clusters in the O. pumila genome in synteny with other MIA-producing plant genomes suggests conserved gene content and gene order (Supplementary Data 25 and 26). Tandem duplications within $O$. pumila MIA gene clusters were also statistically significant, with genes encoding STR, SLS, 7-DLH, 7-DLGT, and other MIA-associated genes being duplicated and gained within identified gene clusters. These gene clusters represent the pangenome for MIA biosynthesis and include several functionally characterized genes, as well as potential genes involved in MIA biosynthesis, which also showed a high correlation with nitrogen-containing metabolites identified in the Ophiorrhiza metabolome (Fig. 7 and Supplementary Figs. 42-48).

\section{Discussion}

The evolution of a specialized metabolite biosynthetic pathway involves the emergence of enzymes that catalyze committed steps toward synthesizing core metabolites. These core metabolites are then subsequently catalyzed by native coopting enzymes, resulting in the colossal chemodiversity of the plant metabolome ${ }^{51,52}$. The expanded chemodiversity, upon serving as a positive selection force, activates the evolutionary machinery, including the emergence of novel specialized enzymes through gene expansion/ neofunctionalization, thus beginning the process of refining the imperfect secondary metabolite biosynthesis pathways ${ }^{53}$. Among Gentianales, the emergence of STR for the synthesis of strictosidine was an important innovation to promote the evolution of MIA biosynthesis, which occurred after the whole-genome triplication of core eudicot genomes (Fig. 6e and Supplementary Fig. 41). While STR-like enzyme homologs were identified and assigned across plant species from different lineages (Supplementary Fig. 29a), functional STRs (OG0015245) were specifically identified in the strictosidine-derived-MIA-producing plants (Fig. 6a and Supplementary Fig. 29b). C. roseus and G. sempervirens genome, which diverged $\sim 68$ Mya from Ophiorrhiza, consisted of a single copy of the STR gene, while the Ophiorrhiza genome contained two STR orthogenes, resulting from tandem duplication. One of the exceptions to the otherwise highly restricted MIA biosynthesis in Gentianales is camptothecin, which was first identified in C. acuminata of Cornales. Similar to the other plant genomes analyzed in this study, C. acuminata lost the functional STR enzyme and did not synthesize strictosidine. Instead, C. acuminata synthesizes strictosidinic acid for the biosynthesis of MIAs, including camptothecin ${ }^{42}$. For C. acuminata, the emergence of a bifunctional SLS (OG0013616) was important for the biosynthesis of strictosidinic acid, which incidentally also showed the fastest rate of substitution among all MIA-producing 


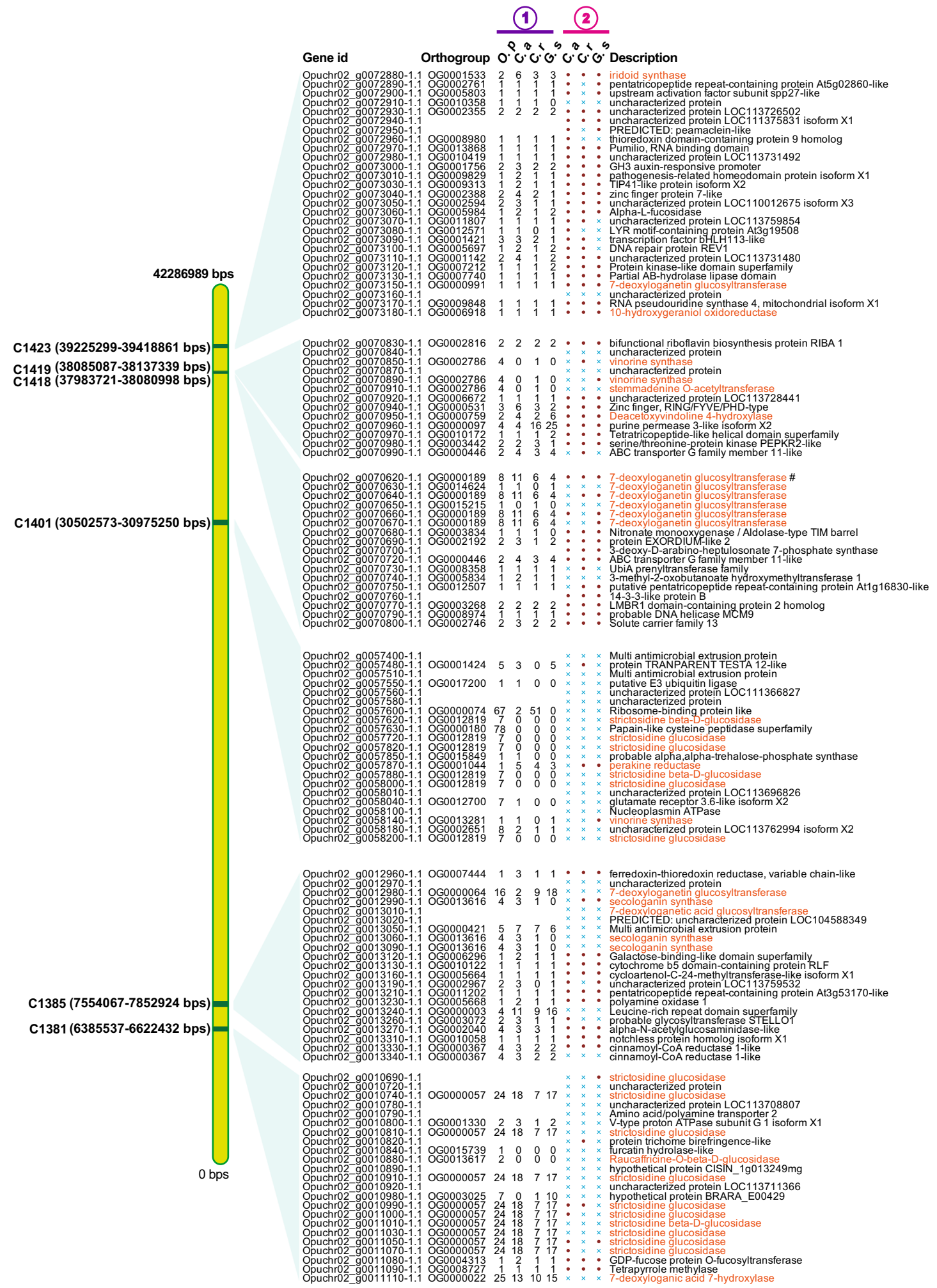

(1) Number of genes assigned to an orthogroup from a specific species

(2) Presence or absence of genes from a syteny relationships with $O$. pumila $\{\cdot$ Present $\times$ Absent $\}$

Fig. 7 Monoterpene indole alkaloid gene clusters identified in chromosome $\mathbf{2}$ in the Ophiorrhiza genome. In total, we identified 33 MIA gene clusters in Ophiorrhiza genome distributed across eight of its chromosomes (Supplementary Figs. 42-48). Conserved synteny at gene clusters across MIA-producing plants suggest role of secondary metabolite clusters toward evolution of specialized metabolites in plants. The position of the gene cluster is scaled based on chromosome size and its physical position on the chromosome. The gene description colored as orange represents genes with functions associated with MIAs biosynthesis. O.p Ophiorrhiza pumila, C.a Camptotheca acuminata, C.r Catharanthus roseus, G.s Gelsemium sempervirens. 
plants (Fig. 6e and Supplementary Fig. 41) ${ }^{43}$. With the WGD peak for C. acuminata detected at peak Ks of 0.469 and median Ks of 0.75 for SLS (OG0013616), our results suggest an earlier emergence of key metabolite intermediates prior to the WGD in C. acuminata, which then served as a catalyst that allowed postWGD expansion and evolution of MIA biosynthesis (Supplementary Fig. 41). Synteny analysis between the coffee and Ophiorrhiza genomes showed significant genome collinearity, yet one of the key enzymes lost in the coffee genome included functional STR orthogene families. The coffee and Ophiorrhiza genomes diverged at $\sim 47 \mathrm{Mya}$, suggesting that while the STR enzyme evolved through SSDs in Ophiorrhiza, the coffee genome instead lost the enzyme required for strictosidine synthesis. Comprehensive metabolite profiling of several species from the Coffea genus, including wild coffee species, could not detect strictosidine, suggesting the possibility that STR has been lost across different species from the Coffea genus ${ }^{54}$. Our study proposes that the retention of STR after the whole-genome triplication event in core eudicots was the critical event that allowed selected plant species to evolve MIAs and expand their chemodiversity (Fig. 6d-f and Supplementary Fig. 41). C. acuminata, the exception, instead uses a promiscuous enzyme for the synthesis of strictosidinic acid, which offered similar opportunities for the evolution of MIA biosynthesis. Despite their similar metabolite intermediates and chemotypes, the two parallel paths to the starting point of MIA biosynthesis in O. pumila and C. acuminata, an estimated speciation time of 120 Mya and their completely different genome dynamics leading to their present-day genomes lead us to propose the possibility of convergent evolution of camptothecin biosynthesis.

As several functional metabolic gene clusters have been reported in the plant genome, identifying and analyzing gene clusters seems to be a promising means to identify candidate genes involved in the biosynthesis of specialized metabolites ${ }^{55}$. Since the number of functionally characterized metabolic gene clusters is still limited in plants, key features such as the extent of coexpression and the degree to which gene localization corresponds to participation within the same pathways are not yet clear. Wisecaver et al., noting that the physical proximity of genes associated with metabolic pathways is statistically significant in Arabidopsis, suggested gene coexpression as a key feature for identifying enzymes associated with known specialized metabolic pathways irrespective of the location of their genes in the genomes $^{49}$. Several studies in the past have also reported the selective nature of coexpression of genes in a predicted metabolic gene cluster $^{39,55-57}$. In the Ophiorrhiza genome, we also observed a lack of coexpression trends among member genes of a given gene cluster. The scattered nature of metabolic gene clusters seems to be prevalent across plant genomes, as observed in the case of MIA gene clusters, as well as previously reported secondary metabolic gene clusters in other plant species ${ }^{55,58}$. With the complexities associated with the regulation of gene-expression in plants, it is only rational to suggest that physical proximity may not be sufficient to facilitate coexpression among genes within a gene cluster $^{57}$. On the other hand, gene clusters represent discrete genome segments that serve as the hotspots for retaining and evolving specialized metabolite biosynthesis. Benzylisoquinoline alkaloid biosynthesis is one of the best-known specialized metabolic pathways, with enzymes forming gene clusters within the opium poppy genome. Nevertheless, the nature of gene clustering was reported to be heterogeneous, with the thebaine and noscapine pathways being highly clustered, and the morphine and sanguinarine pathways being scattered ${ }^{56}$. These results suggest the possibility of the active evolution of genome architecture through a combination of natural and artificial selection for specialized metabolite biosynthesis centered at gene clusters. The gene clusters, therefore, could be regarded as blocks of secondary metabolite modules whose novel combinations could produce new chemotypes, which may offer unique phenotypes for positive selection. In the process of evolution, plants could lose some members of these modules or the entire module itself, and thus also lose the ability to further evolve or refine a particular phenotype. On the other hand, plant species that could retain the specific module could continue to iterate a particular phenotype to more perfectly adapt to the ecological challenges presented over time. As key mechanisms of evolution and speciation, genome restructuring and dynamics acting on gene clusters seems to provide an opportunity to evolve diverge chemotypes across plant species. In this study, we identified the C1541 gene cluster precisely playing this role in strictosidine-derived MIA-producing plants. This implies selection pressure favoring the clusters of genes involved in the biosynthesis of specialized metabolites and could be a way to identify genes involved in the biosynthesis of common metabolite classes going forward. One possible explanation for the positive selection of gene clusters is the reduced rate of recombination between genes involved in local adaptation $^{55,59}$. Positive selection of gene clusters does have a possible role in providing chemotypes that may facilitate ecological/local success for a species or cultivar for successful propagation, as was reported for opium poppy ${ }^{56}$. The conserved nature and collinearity of metabolic gene clusters of the Ophiorrhiza genome across MIA-producing plant species suggest a potential means to select genes for functional studies. The role that gene clusters could play in the divergence of species is not clear, and more high-quality genomes of plants producing specialized metabolites are needed for comprehensive comparative genome analysis to further understand the evolutionary principles that allow a wide distribution of metabolic gene clusters across diverse plant species.

The genome assembly strategy used in this study showed the importance of assembly validation at each step, which should be ignored. Previously, multistage scaffolding was reported as advantageous in assembling the goat genome ${ }^{20}$, and has also been used for other plant genomes $19,21,60$. However, our results showed that the order of scaffolding plays an important role in improved assembly contiguity. One possible explanation for the relevance of the order is the difference in genome resolution for each of the scaffolding technologies. The genome resolution ranges from $30-50 \mathrm{~kb}$ for PacBio reads, $150 \mathrm{~kb}-10 \mathrm{Mb}$ for Bionano optical maps, $\sim 2 \mathrm{Mb}$ for $10 \times$ genomics, and $30-100 \mathrm{Mb}$ for $\mathrm{Hi}-\mathrm{C}^{61}$. Different plant genomes present different challenges, from polyploidy to genome size to repeat content. While we cannot claim that this order of scaffolding technologies will always offer as significant improvement as we observed for the Ophiorrhiza genome, our result certainly showed the importance of assembly validation at each stage of assembly. Stepwise scaffolding and error correction refine the assembly at each stage and therefore assist in achieving high assembly contiguity. While scaffold and contig N50 are widely accepted as genome assembly quality parameters, it is the number of assembly gaps that reflects the real sense of completeness and associated potential misassemblies. Furthermore, although we tested and validated the genome assembly at each stage using multiple orthogonal sequencing technologies, experimental analysis still detected an orientational error in the Ophiorrhiza genome, which suggests that relying solely on sequencing technologies is not sufficient in the pursuit of an accurate genome assembly. The next generation of plant genomes will need to go beyond the construction of mere scaffolds or pseudomolecules and should include experimental validation elements. With the emergence of high-fidelity long reads from PacBio, and longer and more accurate reads from Nanopore, assembly contiguity and contig N50 are expected to 
improve significantly, even for highly heterozygous plant species. Nevertheless, validation steps during and after scaffolding are essential for the accurate interpretation of evolutionary and paleogenomics analysis for all future studies. The status quo of the limited number of near-finished and experimentally validated reference plant genomes needs to change. We believe that this study represents the first step forward in that direction.

\section{Methods}

Plant material and sequencing. For whole-genome sequencing and assembly, we selected O. pumila Champ. ex Benth ${ }^{62}$ (Fig. 3a). O. pumila plants and hairy roots, grown under the aseptic conditions, have been used as a model to investigate biosynthesis of MIA and camptothecin ${ }^{3,12,62-64}$. Genomic DNA for whole-genome sequencing was obtained from the young leaf tissues of 60-day-old O. pumila plant grown in half-strength Murashige and Skoog culture media containing phytoagar (Wako Pure Chemicals, Japan), maintained at $28^{\circ} \mathrm{C}$ in 16 -h day condition in the plant growth chamber 65

Genomic DNA extraction strategies were specific to the sequencing technologies used in this study. For Illumina sequencing, genomic DNA was extracted using Qiagen DNeasy Plant Mini Kit (Qiagen, Hilden, Germany) following the manufacturer's instructions. Extracted genomic DNA was fragmented to an average size of 600 bp with the DNA Shearing System M220 (Covaris Inc., USA). A sequencing library was constructed using the TruSeq DNA PCR-Free Library Prep kit (Illumina, San Diego, USA) and was size-selected on an agarose gel using the Zymoclean Large Fragment DNA Recovery Kit (Zymo Research, CA, USA). The libraries were sequenced on the Illumina HiSeq2500 sequencer in a paired-end mode with a read length of $250 \mathrm{bp}$. High molecular weight genomic DNA for PacBio sequencing was extracted using Qiagen Blood and Cell Culture DNA Mini Kit (Qiagen, Hilden, Germany), following the manufacturer's instructions. Extracted genomic DNA was processed using Qiagen MagAttract HMW DNA Kit (Qiagen, Hilden, Germany) to enrich DNA over $50 \mathrm{~Kb}$ for PacBio sequencing.

For Bionano optical maps sequencing, we used Bionano Prep Plant Tissue DNA Isolation Kit (Bionano genomics, CA, USA), following Bionano Prep Plant Tissue DNA Isolation Base Protocol (part \# 30068). Briefly, $5 \mathrm{~g}$ of fresh young leaves were collected and fixed by formaldehyde treatment followed by homogenization with TissueRuptor (Qiagen, Hilden, Germany). Nuclear fraction was purified with Bionano Prep Plant Tissue DNA Isolation Kit, and the extracted nuclei were embedded in the low-melting agarose plug. Agarose plug was treated with proteinase K (Qiagen, Hilden, Germany) and RNase A (Qiagen, Hilden, Germany) according to the Bionano protocol, and subsequently melted with GELase (Thermo, MA, USA) and dialyzed with TE buffer. Prepared nuclear DNA was labeled using Nb.BssSI (NEB, MA, USA) as restriction enzyme with Bionano Prep Labeling Kit (Bionano genomics, CA, USA), followed by sequencing through Bionano Irys system using one chip, resulting in $101.9 \mathrm{~Gb}$ total data $(>20 \mathrm{~Kb})$ in the form of 8,73,588 molecules. Hi-C library was prepared using Proximo Hi-C plant kit (Phase Genomics, WA, USA) following the manufacturer's protocol with slight modification. In the last step for library preparation, we used a gel extraction-based purification approach to select Hi-C library with a fragment size range of $400-600$ bps. Sau3AI was used as a restriction enzyme to prepare Hi-C libraries. Hi-C libraries were quantified using the Qubit 3.0 Fluorometer (Thermo Fisher Scientific, USA) according to the manufacturer's protocol. We prepared two independent Hi-C libraries and sequenced on the Illumina HiSeq2500 sequencer in the paired-end mode with a read length of $100 \mathrm{bp}$.

Karyotyping and genome size estimation. For $O$. pumila karyotyping, we fixed small flower buds $(<1 \mathrm{~mm})$ without white petals in $3: 1(\mathrm{v} / \mathrm{v})$ ethanol:acetic acid for $24 \mathrm{~h}$ and stored in $70 \%$ ethanol at $4{ }^{\circ} \mathrm{C}$. The preparation of mitotic/meiotic chromosome slides was performed, as previously described with some modifications (Supplementary Fig. 4a, b) ${ }^{66}$. Briefly, after washing the fixed buds in the distilled water, two anthers were digested with $50 \mu$ of enzyme solution containing $4 \%$ cellulase Onozuka RS (Yakult pharmaceutical, Japan) and 2\% pectolyase Y-23 (Kyowa chemicals, Japan) at $37^{\circ} \mathrm{C}$ for $30 \mathrm{~min}$. The digested anthers were transferred to $20 \mu \mathrm{l}$ of $60 \%$ acetic acid solution. Subsequent smearing steps were similar to the procedure described previously ${ }^{66}$. Experiments were repeated twice, and for each experiment, we used at least ten or more chromosome slides.

The genomic size of $O$. pumila was estimated using the flow cytometer approach, and $k$-mer analysis was performed using KmerGenie software ${ }^{67}$ (Supplementary Fig. 4c). Flow cytometer-based analysis was performed, as previously described ${ }^{68}$. Briefly, young leaves of $O$. pumila and A. thaliana were cut using a razor blade in the ice-cold 2-amino-2-(hydroxymethyl)-1,3 propanediol (TRIS)- $\mathrm{MgCl}_{2}$ buffer (0.2 M TRIS- $\mathrm{HCl}, 4 \mathrm{mM} \mathrm{MgCl} 2,0.5 \%$ Triton-X 100, $\mathrm{pH} 7.5$ ), including propidium iodide $\left(50 \mu \mathrm{g} \mathrm{ml}^{-1}\right)$ and ribonuclease $\left(50 \mu \mathrm{g} \mathrm{ml}^{-1}\right)$, and incubated for $5 \mathrm{~min}$. The relative DNA content of isolated nuclei was analyzed using a flow cytometer, FACSCalibur system (Becton Dickinson, New Jersey, USA), while data were acquired and processed by BD FACS DIVA software (v 7.0). No post-fractions were collected, and preliminary FSC/SSC gates for the starting cell population were not used.
De novo genome assembly with parameters optimization. To derive de novo genome assembly using PacBio sequencing reads, we used two assemblers, Canu (v.1.7) ${ }^{22}$ and Falcon-unzip (v.1.3) ${ }^{23}$. We tested multiple parameters specific to the individual assemblers as our effort to optimize and derive best contig-level genome assembly (Supplementary Data 2 and 3). Under different parameters tested for Canu, the best assembly (called Canu-assembly from here on) was used to derive the reference genome assembly (Table 1 and Supplementary Data 2), while best Falcon-unzip assembly (called Falcon-unzip-assembly from here on) was used to derive phased genome assembly of O. pumila (Supplementary Data 3). We used Falcon-unzip-assembly together with Hi-C libraries as the input for the FalconPhase software ${ }^{69}$ to achieve a phased contig-level genome assembly of O.pumila. Briefly, we minced primary contigs in the form of haplotigs pair and collapsed the haplotypes followed with mapping of paired-end $\mathrm{Hi}-\mathrm{C}$ reads to obtain normalized contact matrix. The contact matrix was then used to phase the genome into haplotigs along primary contigs. Default parameters were used for Falcon-Phase software, and the resulting contig-level phased haplotigs of $O$. pumila were used to derive completely phased chromosome-level genome assembly.

De novo genome assembly using Bionano optical map datasets. Acquired Bionano optical maps sequencing datasets were filtered using length cutoff as 150 $\mathrm{Kb}$. We used Canu-assembly as the reference genome to derive guided de novo genome assembly and generated the ".cmap" file. Parameters used for creating ". cmap" files and genome-guided de novo assembly using Bionano optical maps were in accordance with the recommendation of Bionano Solve v 3.0.1 manual (BioNano genomics). Final de novo assembly using Bionano optical maps include 458 scaffolds with N50, and a cumulative assembly length of 1.68 and $442 \mathrm{Mb}$, and over $83 \%$ of optical maps mapped to the Bionano de novo assembly (Table 1).

\section{Chromosome-level genome assembly through stepwise scaffolding. We} adopted a stepwise scaffolding approach to derive the chromosome-scale genome assembly of $O$. pumila. The strategy to derive a high-quality genome assembly included five stages in the order, as described below.

(i) Canu-assembly (or Falcon-unzip-assembly or genome assemblies scaffolded using Hi-C library) was used together with Bionano de novo assembly to derive hybrid scaffolding, using Bionano Solve v 3.0.1 software (BioNano genomics) with default parameters. Hybrid scaffolding detected chimeric sites with conflicts supported by Bionano optical maps and de novo assembly (Supplementary Fig. 3). We inspected conflicts using IrysView software (BioNano genomics), which were manually verified and were used as evidence to split at the sites of misassemblies.

(ii) Canu-Bionano assembly was next split at the gaps and used as input for scaffolding through $\mathrm{Hi}-\mathrm{C}$ libraries. $\mathrm{Hi}-\mathrm{C}$ paired-end reads were aligned to the genome assemblies, using BWA ( $\mathrm{v} 0.7 .16)^{70}$ with strict parameters $(-n 0)$. Read pairs that aligned to different contigs were used for scaffolding.

(iii) After splitting assembly at the gaps of Canu-Bionano-assembly, Hi-C-based scaffolding was derived through the Proximo Hi-C scaffolding pipeline (Phase genomics, CA, USA), as described previously ${ }^{71}$. The proximityguided assembly performed chromosome clustering and determined contig orientations. Briefly, the Proximo Hi-C scaffolding pipeline is based on an enhanced version of LACHESIS algorithm ${ }^{72}$, which additionally performs scaffold optimization and quality control steps based on interaction probabilities to group and orient contigs. Hi-C interactions binned the contigs into 11 groups (corresponding to the haploid or phased chromosomes) and successfully oriented all contigs. The gap size between the ordered contigs was set to $25 \mathrm{bp}$. Canu-assembly, scaffolded through Bionano followed by Hi-C, was further checked for the presence of any assembly conflicts and was thoroughly verified, using $\mathrm{Hi}-\mathrm{C}$ reads-based contact matrix, Bionano raw reads, and Bionano de novo assembly (Fig. 3b-1, and Supplementary Figs. 8 and 9).

(iv) The final genome assembly was subjected to PacBio reads-based gap filling, using PBJelly ${ }^{73}$ from PBSuite v15.8.24 with default parameters. PbJelly closed 64 out of the 85 assembly gaps.

(v) We next performed assembly polishing. Firstly, we performed three rounds of assembly polishing by PacBio reads, using arrow software (https://github. com/PacificBiosciences/GenomicConsensus/tree/develop/

GenomicConsensus/arrow). Arrow-based polishing was followed by a final round of error correction, using Illumina reads through Pilon software ${ }^{74}$. Illumina sequencing reads were trimmed based on Phred score using Trimmomatic software ${ }^{75}$, mapped to the genome assembly using Bowtie $2.0^{76}$, and were subsequently used for Pilon software-based error correction.

For in tandem scaffolding, assemblies scaffolded using Bionano datasets or Hi$\mathrm{C}$ were first disintegrated at the assembly gaps and were subsequently used as input for scaffolding by either by $\mathrm{Hi}-\mathrm{C}$ or Bionano datasets, respectively (Table 1). The final reference and phased genome assemblies of $O$. pumila were validated based on Bionano optical maps, Bionano de novo assembly using IrysView software, and HiC contact map using Juicerbox software ${ }^{77}$ (Fig. 2b-l, and Supplementary Figs. 8 and 9). BioNanoAnalyst ${ }^{78}$ software-based assembly quality assessment showed 
$100 \%$ of the assembly supported by Bionano optical maps for O. pumila genome assemblies.

\section{Phasing genome assemblies into haplotigs. We used contig-level genome} assemblies, obtained from analyzing Falcon-unzip-assembly with $\mathrm{Hi}-\mathrm{C}$ reads using Falcon-Phase software, to derive phased genome assemblies of O. pumila (Supplementary Data 3 and 4). Each of the contig-level haplotigs was first scaffolded using Bionano de novo assembly followed by Hi-C-based scaffolding. Subsequently, PbJelly-based gap filling and assembly polishing were performed, as described for O. pumila reference genome assembly (Supplementary Data 4). Comparing O. pumila-phased genomes with the reference genome assembly showed perfect alignment except an assembly gap in chromosome 1 of the hap lotigs (Supplementary Fig. 5), which originates from the difference between contiglevel assembly, resulting from Falcon-unzip and Canu, the primary assemblers used.

Experimental validation of genome assembly. We first identified repeats in $O$ pumila genome and masked repeats using RepeatMasker (http://www. repeatmasker.org/RMDownload.html), and fragmented the genome assemblies along chromosomes into 20 or $40 \mathrm{~Kb}$ genome segments. We performed BLASTNbased analysis using each fragment as query against all genome fragments together as the database. The genome segments with $\leq 2 \%$ identity were regarded as nonhomologous genomic regions and the repeat-free nonhomologous regions $>7 \mathrm{~Kb}$ adjacent to the genome assembly gaps were selected as probes. PCR primers were designed using Primer 3 (http://bioinfo.ut.ee/primer3/) (Supplementary Data 6). Touchdown PCR with KOD Plus Neo (TOYOBO, Japan) was conducted as follows: $94^{\circ} \mathrm{C}$ for $2 \mathrm{~min}$, five cycles of $94^{\circ} \mathrm{C}$ for $15 \mathrm{~s}$ and $74^{\circ} \mathrm{C}$ for $6 \mathrm{~min}$, five cycles of $94^{\circ} \mathrm{C}$ for $15 \mathrm{~s}$ and $72^{\circ} \mathrm{C}$ for $6 \mathrm{~min}$, five cycles of $94^{\circ} \mathrm{C}$ for $15 \mathrm{~s}$ and $70^{\circ} \mathrm{C}$ for $6 \mathrm{~min}$, and 30 cycles of $94^{\circ} \mathrm{C}$ for $15 \mathrm{~s}$ and $68^{\circ} \mathrm{C}$ for $6 \mathrm{~min}$. Purified PCR products, showing a single DNA band on electrophoresis, were labeled by DIG-Nick Translation Mix (Sigma-Aldrich, MO, USA). For FISH analysis, $10 \mu \mathrm{l}$ of hybridization solution ( $50 \%$ formamide, $10 \%$ dextran sulfate, $2 \times$ SSC, $200 \mathrm{ng}$ of each probe) was applied to each chromosome slide, covered with $22 \times 22 \mathrm{~mm}$ coverslip and sealed with a paper bond (Kokuyo), and the chromosomal DNA and the probe DNA were denatured for $4 \mathrm{~min}$ using a heat block $\left(80^{\circ} \mathrm{C}\right)$. The slide was incubated in a moisture chamber at $37^{\circ} \mathrm{C}$ for 2 days. After washing in the distilled water, $125 \mu$ lof the antibody cocktail ( $1 \%$ BSA (Roche), $4 \times$ SSC, $0.1 \mu$ g anti-digoxigenin-rhodamine Fab fragments (Roche)) was applied and covered with parafilm, and incubated in a moisture chamber for $60 \mathrm{~min}$ at $37^{\circ} \mathrm{C}$. The chromosome slide was air-dried after washing it three times in $42^{\circ} \mathrm{C}$ distilled water each time for $5 \mathrm{~min}$. Finally, we counterstained chromosomes with $5 \mu$ l of VECTASHIELD (Vector Laboratories) containing $5 \mu \mathrm{g} \mathrm{ml}^{-1}$ of 4, 6 diamidino-2-phenylindole (Thermo Fisher Scientific). FISH signals were captured with an OLYMPUS BX-53 fluorescence microscope equipped with a CCD camera (Photometrics Cool SNAP MYO), and processed by MetaVue/MetaMorph (v.7.8) and Adobe Photoshop CC. Image J (https://imagej. nih.gov/ij/) was used to straighten pachytene chromosomes. For experimentally validating the orientations of contigs within a scaffold, the position of FISH probes was compared with the expected position along the chromosome arms. We also used repeat analysis to identify the pericentromeric region and centromere repeats annotated as putative OpuCEN (Supplementary Fig. 16). C. arabica, an allotetraploid genome resulting from hybridization between C. canephora and C. eugenioides, was recently sequenced ${ }^{79,80}$. We designed FISH probes corresponding to the site of potential misassembly in the C. canephora genome and tested in $C$. arabica. FISH analysis for $C$. arabica was performed as has been described for $O$. pumila, and fresh roots were chosen for the experiment. FISH analysis for all probes were performed as two independent experiments, and for each probe, the signals were confirmed for at least ten or more instances.

Genome assessment. The $O$. pumila genome assembly and phased genome assemblies were benchmarked using BUSCO $(v 3.0 .2 \mathrm{~b})^{30}$. We identified 1335 out of $1375(97.1 \%)$ complete gene models and six fragmented gene models $(0.4 \%)$ in $O$. pumila reference genome assembly; $95.0 \%$ of these complete gene models were single copy, while only $2.1 \%$ had more than one copy (Fig. 3c). BUSCO analysis for O. pumila-phased genome assemblies (haplotigs) identified $91.2-91.4 \%$ of core gene models. We compared predicted gene models with de novo transcriptome assembly derived using RNA-seq datasets for five tissues and previously published datasets of O. pumila ${ }^{3,15,65}$. De novo transcriptome assembly was derived using Trinity software (v 2.6.6) ${ }^{81}$. Mapping these unigenes to the O. pumila genome using BLAT software ${ }^{82}$ showed $99.32 \%$ of the unigenes could be identified, suggesting a good representation of coding sequences in the genome.

Gene prediction and functional annotation. Gene models for O. pumila genome assembly were predicted as described before ${ }^{83}$. Briefly, evidence-based gene prediction was first performed by BRAKER 2 software ${ }^{84}$, using O. pumila de novo transcriptome assembly. The predicted gene models together with gene models of C. canephora $(\mathrm{v} \mathrm{1.0})^{29}$, Nicotiana tabacum ${ }^{85}$, and Trifolium pratense $(\mathrm{v} 2.0)^{86}$, were used as training sets for the ab initio gene prediction. Gene model prediction was performed using the MAKER-P pipeline ( $\mathrm{v} 2.31 .8)^{87}$ by incorporating three ab initio gene prediction tools, namely, AUGUSTUS (v 3.3) ${ }^{88}$, SNAP (v 2006-07-28) ${ }^{89}$, and GeneMark_ES (version 4.33) ${ }^{90}$. In parallel, we performed an evidence-based gene model prediction by mapping $O$. pumila transcriptome datasets onto the assembled genome sequences, using TopHat (v 2.1.1) and Cufflink (v 2.2.1) pipeline ${ }^{91}$. The predicted gene models were used to perform InterProScan ${ }^{92}$ against the InterPro database and BLAST search against GyDB $2.0^{93}$ with an E-value cutoff as 1.0. Gene models annotated as TEs based on GyDB 2.0 or InterProScan annotation were excluded for analysis, and regarded as de novo predicted novel TEs of O. pumila genome. The remaining gene models were subjected to homology searches against NCBI-nr database, A. thaliana in TAIR11, and SwissProt protein databases using BLASTP with an E-value cutoff of $1 \mathrm{E}-20$. The gene models with homology against searched databases or annotation edit distance (AED) score $\leq 0.9$ were selected as high-confidence intrinsic gene models, resulting in 32,389 gene models for the O. pumila genome in total. The rest of the predicted gene models with no RNA-seq evidence or annotation were categorized as low-quality gene models and were not used for any downstream analysis. Annotated gene models were functionally mapped and annotated using OmicsBox software (BioBam). Annotation-based GO terms assigned to $O$. pumila gene models showed the top six assigned biological processes related to different metabolic processes, including organic substance metabolic process and nitrogen compound metabolic process (Supplementary Fig. 10f). O. pumila gene models were also assigned to TFs families based on PlantTFDB classification ${ }^{94}$. The TF database was obtained from PlantTFDB (http://planttfdb.cbi.pku.edu.cn/ download.php) and used for reciprocal best hit (RBH), using blast_rbh.py script (https://github.com/peterjc/galaxy_blast/tree/master/tools/blast_rbh). A total of 778 genes were assigned to $54 \mathrm{TF}$ families, including bHLH, MYB, NAC, C2H2, and ERF based on the number of assigned genes (Supplementary Data 7).

O. pumila noncoding RNAs were annotated using multiple databases and software packages. The tRNA genes and their secondary structure were identified by tRNAscan-SE software ${ }^{95}$ with default parameters (Supplementary Data 9). The ribosomal RNAs (rRNAs) were predicted based on BLASTN search against rRNA sequences at an E-value cutoff of $1 \mathrm{e}-10$. For microRNAs and small nuclear RNA (snRNA) coding genes prediction, we used INFERNAL software ${ }^{96}$ against the Rfam database (release 13) ${ }^{97}$. In total, we identified 90 miRNAs and 2032 snRNAs in the O. pumila genome (Supplementary Data 8).

De novo transposable elements and repeat annotation. We used known repetitive sequences in Repbase (http://www.girinst.org/repbase/) ${ }^{98}$ and de novo repeat libraries to annotate $O$. pumila repeat contents. For the de novo repeat prediction, we used RepeatModeler (v 1.0.11) (http://www.repeatmasker.org/ RepeatModeler/), LTR_FINDER ${ }^{99}$, and RepeatScout $1.0 .5^{100}$. The repetitive elements in the Repbase and $O$. pumila de novo repeat library were annotated using RepeatMasker (v 4.0.7) $)^{101}$. Tandem repeats of the O. pumila genome were identified using Tandem Repeats Finder (TRF) software ${ }^{102}$. Using TRF, we identified telomere regions for all eleven chromosomes of O. pumila (Supplementary Data 11).

Whole-genome duplication and intergenomic analysis. To understand O. pumila genome evolution, we searched for genome-wide duplications in the assembled $O$. pumila genome. We performed the self-alignment of the genome assembly using LAST (v 963) ${ }^{103}$. Using a cscore filter of 0.7 , we filtered LAST-run output to identify significantly matching sequences within the O. pumila genome. The analysis suggested a minimal genome duplication within the O. pumila genome, with 2917 gene sets showing small stretches of duplication in the form of 200 clusters (Supplementary Fig. 10b). MCSCANX ${ }^{104}$ based synteny analysis of O. pumila genome with default parameters detected 132 syntenic blocks representing 3227 genes ( $9.97 \%$ of genomic space), while 1351 gene pairs were identified as tandem repeats. Minimal segmental duplication blocks suggested small-scale background duplications rather than a WGD event in O. pumila.

We next compared $O$. pumila genome assembly with 12 other plant genomes,

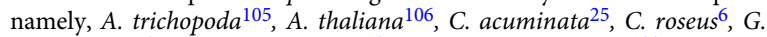
sempervirens ${ }^{6}$, Helianthus annuus ${ }^{107}$, Lupinus angustifolius ${ }^{108}, N$. benthamiana ${ }^{31}$, P. somniferum ${ }^{109}$, S. lycopersicum ${ }^{32}$, and V. vinifera ${ }^{27}$. We identified paralogs by all_vs_all BLASTP search for each of the plant genomes with E-value cutoff as le -10 , followed by $\mathrm{MCL}^{110}$ clustering with inflation factor 1.5. Identified paralog groups with a maximum of 100 genes were selected to perform pairwise sequence alignment using MUSCLE (v 3.8.31) ${ }^{111}$, with the number of times to perform ML estimation set as 5 . The synonymous substitution rate (Ks) for paralogous gene pairs were calculated using codeml program of PAML package ${ }^{34}$. The Ks distribution plot for O. pumila paralogs showed a typical gamma $(\gamma)$ event corresponding to whole-genome triplication, but no peak corresponding to a new WGD event was identified (Fig. 3e and Supplementary Fig. 19). Similar to O. pumila, no WGD was detected in C. roseus. For C. acuminata, Ks distribution plot clearly showed two peaks at Ks value 1.681 , representing eudicot wholegenome triplication, and 0.469 , representing a recent whole-genome duplication (Supplementary Fig. 19). Using whole-genome triplication time as $\sim 154 \mathrm{Mya}$ and Ks-peak as 1.681 , we estimated substitution rate as $5.547 \times 10^{-9}$ mutations per site per year $(r)$ for $C$. acuminata. Thus, the whole-genome duplication time for C. acuminata was dated as $42.27 \pm 0.73$ Mya using the formula $T=\mathrm{Ks} / 2 r$ (where standard deviation was calculated based on Ks standard deviation of C. acuminata paralogs centered around the Ks median value; Supplementary Figs. 18 and 19). We next compared the O. pumila genome with 12 plant species by first identifying 
RBH using blast_rbh.py script between O. pumila and other plant species, and then performing pairwise sequence alignment using MUSCLE (v 3.8.31) ${ }^{111}$ with the number of times to perform ML estimation set as 5 . The Ks values for RBHs of O. pumila with other plant species were calculated using the codeml program of the PAML package $^{34}$ (Supplementary Fig. 20).

Phylogenetic tree reconstruction and divergence time prediction. The assembled genome of $O$. pumila allowed us to understand its evolution and to estimate divergence time within Rubiaceae species. In order to achieve a robust phylogenetic reconstruction with high confidence and concordance, we used gene models for 33 plant species; namely, A. trichopoda ${ }^{105}$, Aquilegia coerulea ${ }^{112}$, A. thaliana $a^{106}$ Brachypodium stacei (B. stacei v 1.1 DOE-JGI, http://phytozome.jgi.doe.gov/), Brassica rapa ${ }^{113}$, C. acuminata ${ }^{25}$, C. roseus ${ }^{6}$, Cicer arietinum ${ }^{114}$, Citrus clementina $^{115}$, C. canephora ${ }^{29}$, Cucumis sativus ${ }^{116}$, G. sempervirens ${ }^{6}$, Glycine max ${ }^{117}$, Glycyrrhiza uralensis ${ }^{118}$, Gossypium raimondii ${ }^{119}$, H. annuus ${ }^{107}$, L. angustifolius ${ }^{108}$, Malus domestica ${ }^{120}$, Medicago truncatula ${ }^{121}$, Musa acuminata ${ }^{122}$, Nelumbo nucifera $^{123}$, N. benthamiana ${ }^{31}$, O. pumila (this study), Oryza sativa ${ }^{124}$, P. somniferum $^{109}$, Populus trichocarpa $a^{125}$, Prunus persica ${ }^{126}$, Selaginella moellendorffii ${ }^{127}$, S. lycopersicum ${ }^{32}$, Sorghum bicolor ${ }^{127}$, Theobroma cacao ${ }^{128}$, V. vinifera $^{27}$, and Zea mays $^{129}$; thus covering diverse plant lineages. Using OrthoFinder (v 2.3.1) ${ }^{33}$, we identified 31 single-copy orthologous genes from these selected plant genomes. Single-copy genes for each plant species for a given orthogroup were aligned using MUSCLE (v 3.8.31) ${ }^{111}$, and alignments were concatenated to create a super alignment matrix. The concatenated alignment was subsequently used to construct a maximum likelihood phylogenetic tree using RAxML (v 8.2.11) ${ }^{130}$. The concatenated alignment was subsequently used to construct a maximum likelihood phylogenetic tree using RAxML (v 8.2.11).

The derived phylogenetic tree was used to infer divergence time using the MCMCtree program ${ }^{34,131}$ implemented in the phylogenetic analysis by maximum likelihood, using S. moellendorffii as an outgroup. The MCMCtree analysis was executed using the following parameters: burn-in-10,000, sample-frequency-2, sample number-100,000. For the divergence time estimation, we calibrated the model using divergence time between O. sativa and A. thaliana (148-173 Mya), $B$. stacei and O. sativa (42-52 Mya), A. thaliana and V. vinifera (105-115 Mya), and $S$. moellendorffi and A. trichopoda (410-468 Mya), obtained from the TimeTree database ${ }^{132}$. The time of speciation between C. canephora and O. pumila was estimated as 47 Mya (Supplementary Fig. 18). Using the divergence time and Kspeak between $C$. canephora and $O$. pumila, we estimated synonymous substitutions per site per year $(r)$ for Rubiaceae as $6.54 \mathrm{e}-9(T=\mathrm{Ks} / 2 r)$.

Expansion and contraction of gene families. Protein sequences of $O$. pumila and 12 other plant species, namely, A. trichopoda, A. thaliana, C. acuminata, C. roseus, C. canephora, G. sempervirens, H. annuus, L. angustifolius, N. benthamiana, P. somniferum, S. lycopersicum, and V. vinifera were used for the gene family construction. We filtered sequences of length $<30$ amino acids and performed CDHIT-EST ${ }^{133}$-based protein clustering to select the longest sequences from the cluster of highly similar sequences for a species. Protein sequences for all the plant species were used as input and were grouped in orthogenes families using Orthofinder ( $\mathrm{v}$ 2.3.1) $)^{33}$ using the following parameters: $-\mathrm{S}$ blast $-\mathrm{t} 70-\mathrm{M}$ msa $-\mathrm{A}$ muscle $-\mathrm{T}$ raxml-ng -I 1.5. In total, 18,226 orthogroups were assigned across 13 plant species, including 675 single-copy genes, with 23,229 genes of $O$. pumila being assigned to the orthogroups (Supplementary Data 20). We identified 95 orthogroups representing 632 genes specific to O. pumila, while 514 orthogene families were specific to MIAs producing plant species (O. pumila, C. acuminata, G. sempervirens, and C. roseus) with 1,078 genes from O. pumila genome and 2,885 genes across the MIA-producing plant species.

We estimated orthogene family gain, expansion, loss, and contraction by comparing the cluster size between species, using COUNT software (Fig. 5) ${ }^{134}$. We first derived the maximum likelihood phylogenetic tree using 675 single-copy orthologs across 13 plant species used for the gene family construction, as described above. The definition of orthogene family evolution in terms of gains, expansions, losses, or contractions are described, using a posterior probability in the COUNT software user's guide (http://www.iro.umontreal.ca/ csuros/ gene_content/count-usage.pdf). COUNT software reconstructs ancestral state using orthogenes family count data and phylogeny relationships and compares the closest outgroup. It uses phylogenetic birth-and-death models for the probabilistic inferences, where rates are optimized using the selected orthogenes family size. For the rate optimization, we used the gain-loss-duplication model type with Poisson distribution for the family size at the root of the phylogenetic tree, and the same gain-loss and duplication-loss ratio was selected as a lineage-specific variation. The maximum number of optimizations rounds for COUNT software was set as 10,000, and the convergence threshold of the likelihood was set as 0.1. Optimized rates for the evolution of orthogenes families were used to calculate family history by posterior probabilities, which provided the corresponding $p$ values in each lineage (Supplementary Data 21). A $p$ value of 0.05 was used to identify gene families gained/expanded/lost/contracted at a specific lineage or species. Orthogroups specifically gained or expanded in plants producing MIAs were further investigated centered around $O$. pumila.
Isotope labeling-based approach to identify nitrogen-containing metabolites Using a ten-step cheminformatics workflow, we performed nitrogen number determination, chemical formula prediction, and structure elucidation for unknown metabolites based on complete nitrogen labeling and liquid chromatography-tandem mass spectrometry (LC-MS/MS) approach, as previously described ${ }^{7}, 40$. We performed complete stable isotope nitrogen labeling $\left({ }^{15} \mathrm{~N}\right)$ for $O$. pumila metabolome pool using hairy roots to capture diverse nitrogen-containing specialized metabolites (i.e., MIAs). O. pumila hairy roots were maintained as previously described ${ }^{65}$. For complete nitrogen labeling, we replaced $\mathrm{KNO}_{3}$ and $\left(\mathrm{NH}_{4}\right)_{2} \mathrm{SO}_{4}$ with complete nitrogen-labeled substitutes $\left({ }^{15} \mathrm{~N}\right)$ in the growth media, while control samples were maintained in non-labeled nitrogen sources (Supplementary Data 13). Two generations of isotope labeling were used to dilute any carryover from the starting material and to achieve a high degree of ${ }^{15} \mathrm{~N}$ labeling. Hairy root, post isotope labeling, was used for metabolites extraction and profiling using LC-Q-TOF/MS (LC, Waters Acquity UPLC System; MS, Waters Xevo G2 QTOF). The method used for metabolite extraction, purification, and metabolite profiling, including UHPLC conditions, the column used, and MS and MS/MS conditions, have been previously described ${ }^{40}$. Metabolite profiling datasets were analyzed to evaluate the extent of nitrogen labeling of the metabolome space. Principal component analysis clustered samples as two clear groups based on control or nitrogen labeling (Supplementary Fig. 21). We next selected metabolite features distinguishing isotope-labeled vs non-isotope-labeled samples using loadings plot and S-plot. For these selected metabolite features, chemical structures and the number of nitrogen atoms were identified using cheminformatics approach previously described ${ }^{40,135}$. Identified metabolites were manually validated based on mass shift expected due to stable isotope labeling of ${ }^{15} \mathrm{~N}$, as well as to exclude any metabolites that were assigned structures due to contamination through in-source fragmentation. Identified metabolites were next compared to previously reported metabolome data for 11 other plant species (Fig. 4$)^{40}$. We also performed metabolite profiling for the same five tissues and O. pumila hairy roots that were used to perform transcriptome profiling (Supplementary Figs. 22 and 23, and Supplementary Data 15). Metabolite profiling for isotope-labeled hairy roots, and tissues of $O$. pumila was performed using five biological replicates, and a newly established metabolome database for $O$. pumila was used to assign chemical identity for metabolites and to perform differential metabolome analysis.

RNA preparation and expression analysis. To facilitate gene model prediction and to capture diverse genes expression, we extracted RNA from five tissues of $O$. pumila, namely, root, stem, shoot-apex, internode, and leaf. Total RNAs from these tissues were extracted using RNeasy Plant Mini Kit (Qiagen, USA) following the manufacturer's protocol. The RNA quality was assessed using Agilent Bioanalyzer 2100 (Agilent Technology, USA), and RNA samples with RNA integrity number $(\mathrm{RIN})>8.0$ were used for cDNA library preparation. Illumina libraries for RNA sequencing were prepared, as previously described ${ }^{136}$. cDNA libraries for Illumina sequencing were prepared using the SureSelect Strand-specific RNA library kit (Agilent Technology, USA) according to the manufacturer's instructions, and sequenced using Illumina HiSeq 2000 sequencer (Illumina, San Diego, USA) to obtain paired-end reads with an average length of $101 \mathrm{bp}$ and a total of $10 \mathrm{~Gb}$ sequencing reads for each of the five tissues. For gene model prediction, we used RNA-seq datasets for five tissues generated in this study, and datasets previously acquired from our laboratory deposited under DDBJ accession nos.-DRA000930, DRA000931, and SRA492327. Transcriptome profiling datasets for hairy roots and cell suspension culture of $O$. pumila were obtained from DDBJ (accession no. DRA000930). RNA-seq raw reads were processed to remove adapters and poorquality reads (base quality score $>30$ ) using Trimommatic software $(\mathrm{v} 0.38)^{75}$. The processed reads were used as an input for Trinity software ( $\mathrm{v} 2.6 .6)^{137}$ to derive de novo transcriptome assembly, which was then used as EST evidence to validate $O$. pumila gene model prediction. For expression analysis, we used Tophat2 software (v 2.1.1) $)^{91}$ to map clean RNA-seq reads to O. pumila reference genome with the following parameters: -max-intron-length 500000, -read-gaplength 10,-read-edit-dis 15, -max-insertion-length 5; -max-deletion length 5 . The expression level for $O$. pumila genes (RPKM, TPM, and expression count data) was obtained using HTSeq software (v 0.11.1)

We adopted a targeted approach to identify high-confidence candidate genes associated with MIAs biosynthetic pathway. We first manually curated genes that have been functionally characterized to be associated with MIAs biosynthesis pathways, accounting for 94 genes in total (Supplementary Data 16). Protein sequences for these genes were used as a database, and O. pumila gene models were annotated through this database using BLASTP with E-value cutoff as $1 \mathrm{E}-20$ and an alignment length over 100 amino acids. In total, 1226 O. pumila genes were annotated as putative genes sharing high sequence similarity with functionally characterized genes (Supplementary Data 17). We next performed CD-HIT-ESTbased protein sequence clustering using all $O$. pumila gene models and 94 genes from MIAs protein database. Protein clusters that included O. pumila genes together with functionally characterized MIAs genes were selected. CD-HIT-ESTbased protein clustering and BLASTP-based annotation were used to select highconfidence genes associated with MIAs biosynthesis in O. pumila. We selected 216 $O$. pumila genes representing 40 known enzymes involved in the biosynthesis of MIAs (Supplementary Data 18). These genes were used as criteria to identify MIAs gene clusters 
We next performed coexpression analysis using genes assigned to the secoiridoid biosynthesis branch of MIAs biosynthesis pathways. Coexpression analysis, using Spearman's correlation followed by hierarchical clustering, identified a highly coexpressed gene cluster representing the complete secoiridoid biosynthetic pathway, including all four functionally characterized genes of $O$. pumila associated with MIA biosynthesis (Supplementary Fig. 24). These genes were used to perform coexpression analysis with other $O$. pumila genes assigned to MIAs biosynthetic pathway, and a highly coexpressed gene cluster was selected (Supplementary Figs. 1 and 25). Heat maps to visualize the expression of assigned MIAs biosynthesis genes were drawn using a heatmap2.0 package $^{139}$ in $\mathrm{R}(\mathrm{v}$ 3.5.3), while coexpression analysis and hierarchical clustering was performed using inbuilt functions in R. For integrative omics analysis and gene-metabolite correlation network, we used normalized expression dataset (in the form of transcript per million) for genes assigned to secoiridoids and MIA biosynthesis pathways with metabolome datasets (normalized using internal standard), and performed pairwise Pearson's correlation analysis using in-built psych package (https://cran.r-project. org/web/packages/psych/index.html) in R. Edges between genes and metabolites are drawn using Cytoscape v 3.6.1, when the correlation coefficient between genes and metabolites are $>0.7$ with a corrected $p$ value $<0.05$.

Metabolic gene cluster prediction. For gene cluster analysis, we used PlantClusterFinder software (v 1.3) pipeline ${ }^{140}$. We used O. pumila gene models to assign four-part EC numbers and MetaCyc reaction identifiers based on protein sequence data, and classification according to the predicted catalytic functions by E2P2 software (v 3.1) ${ }^{141}$. E2P2-based enzyme annotation assigned $9584 \mathrm{O}$. pumila genes with an EC number, which were then converted into the corresponding MetaCyc (v 22.5) ${ }^{142}$ reaction identifiers. It was used for pathway inference and pathway database construction using the PathoLogic software (v 22.5) ${ }^{143}$ (Pathway Tool software). The derived pathways database was then manually curated and validated, using SAVI software (v 3.0.2) ${ }^{140}$ to remove any false positive and redundant pathways, such as non-plant pathway variants, and pathways already included as part of a larger pathway. The pathway database for O. pumila with assigned metabolic reactions and enzymes were then used as input together with $O$. pumila genome annotation structure for the PlantClusterFinder software, as instructed by the tool developers.

Using the PlantClusterFinder pipeline, we identified 358 gene clusters representing 3387 genes of the O. pumila genome (Supplementary Data 23 and 24). To assign gene clusters to MIA biosynthesis, we mapped $O$. pumila genome annotation using MIAs protein database and considered a gene cluster as an MIA gene cluster if it included one or more of the 216 high-confidence MIAs biosynthesis genes. We identified 33 MIA gene clusters across 8 out of the 11 chromosomes (Fig. 7 and Supplementary Figs. 42-48). The obtained gene clusters were mapped with synteny data to compare the $O$. pumila genome with three other MIAs producing plants, namely, C. acuminata, C. roseus, and G. sempervirens (Fig. 7, Supplementary Figs. 42-48, and Supplementary Data 23-26). Despite the fragmented genome assemblies for other MIA-producing plants, synteny analysis showed conserved gene order centered at $O$. pumila MIAs gene clusters to be statistically significant based on one-sided Fisher exact test.

\section{Divergence time estimation of orthogene families and syntenic metabolic}

gene clusters. The phylogenomic analysis was performed to estimate divergence time for syntenic genes that were part of secondary metabolite gene clusters. We first selected syntenic gene pairs between $O$. pumila and other three MIAs producing plants, namely, C. acuminata, C. roseus, and G. sempervirens, as well as $C$. canephora, another plant from Rubiaceae family. Syntenic gene pairs, including a member of one of the 358 metabolic gene clusters identified in the O. pumila genome, were selected for further analysis. For each pair, which is also a member of a metabolic gene cluster in the O. pumila genome, protein sequence alignment for corresponding syntenic genes from the selected plant genomes was performed using MAFFT ${ }^{144}$, and Ks values were calculated as described above (Supplementary Data 25 and 26). Median Ks value for a gene cluster block was estimated, and was considered as Ks value for that specific gene cluster with respect to the plant species that were used for comparison. We then used one-sided Fisher's exact test to calculate statistical significance for divergence time of MIA gene clusters, using syntenic analysis data of the whole genome of $O$. pumila as a reference set. For Ks analysis of genes assigned to a given orthogene family, we performed pairwise alignment for paralogs of a given plant species for an orthogene ID, and Ks values for pairwise alignment were estimated, as described above (Fig. 6e, Supplementary Fig. 41, and Supplementary Data 22). For the calculation of Ks median, we discarded values with alignment length $<300 \mathrm{bp}$ and alignment coverage $<0.2$.

We used tools from the ete 3 pipeline ${ }^{145}$ to generate maximum likelihood phylogenetic trees for orthogene families using MUSCLE as aligner, trimal_gappyout as alignment cleaner, pmodeltest_soft_slow as model tester, and raxml:default_bootstrap as tree builder (Supplementary Figs. 30-40). We also performed BUSTED analysis ${ }^{48}$ to ascertain if the STR or SLS clade, including orthogenes, gained only in MIA-producing species, as well as conserved across other plant species, has experienced positive selection for at least one site and at least one branch, with $p$ level cutoff set as 0.05 for significance (Fig. 6b, d). For BUSTED analysis, we used orthogroups specific to MIA-producing plants as a query, and rest of the genes in the phylogenetic tree were used as the background.
Reporting summary. Further information on research design is available in the Nature Research Reporting Summary linked to this article.

\section{Data availability}

The datasets and plant materials generated and analyzed during the current study are available from the corresponding author upon request, and subjected to the material transfer agreement. All raw genome sequencing datasets have been deposited at the DDBJ database (Experiment: DRX185163-DRX185191; Run: DRR194711-DRR194739) under the accession id-DRP006713; with BioProject id-PRJDB8685, submission idDRA009076. Assembled O. pumila genome assembly has been deposited at the DDBJ database (accession ids-BLIW01000001-BLIW01000013). All sequence datasets, assembled genome sequences, predicted gene models, transcriptome datasets, genome browser, annotation, and KEGG mapping results are available to download and analyze through a dedicated server to perform comparative genome analysis (http://pumila. kazusa.or.jp/). RNA-seq datasets have been deposited under submission accession id DRA011108 (https://www.ncbi.nlm.nih.gov//sra/?term=DRP006713/rna)under the experiment accessions DRX245380-DRX245384, and sample accessions DRS163600-DRS163604. For gene model prediction, we also used RNA-seq datasets previously acquired from our laboratory and deposited under DDBJ accession nos.DRA000930, DRA000931, and SRA492327. Transcriptome profiling datasets for hairy roots and cell suspension culture of $O$. pumila were obtained from DDBJ (accession no DRA000930). Data supporting the findings of this work are available within the paper and its Supplementary Information files. All results and generated datasets, including comparative genome analysis, transcriptome analysis, metabolome analysis, and integrative omics analysis are available in the form of supplementary datasets. A reporting summary for this article is available as a Supplementary Information file. Source data are provided with this paper.

\section{Code availability}

Codes for hybrid genome assembly, and the RScript used to create Fig. 4 together with the comparative metabolome dataset and associated metabo-ontologies are available through GitHub (https://doi.org/10.5281/zenodo.4279079)146. Parameters used for genome assembly, annotation, comparative genome analysis, and metabolome analysis have been provided in detailed Supplementary Methods and Supplementary Tables in the relevant sections.

Received: 16 July 2020; Accepted: 7 December 2020; Published online: 15 January 2021

\section{References}

1. Dagenais, G. R. et al. Variations in common diseases, hospital admissions, and deaths in middle-aged adults in 21 countries from five continents (PURE): a prospective cohort study. Lancet 395, 785-794 (2020).

2. De Luca, V. Monoterpenoid Indole Alkaloid Biosynthesis (Wiley, New York, 2011).

3. Udomsom, N. et al. Function of AP2/ERF transcription factors involved in the regulation of specialized metabolism in Ophiorrhiza pumila revealed by transcriptomics and metabolomics. Front. Plant Sci. 7, 1861 (2016).

4. Qu, Y. et al. Solution of the multistep pathway for assembly of corynanthean, strychnos, iboga, and aspidosperma monoterpenoid indole alkaloids from 19E-geissoschizine. Proc. Natl Acad. Sci. USA 115, 3180-3185 (2018).

5. Miettinen, K. et al. The seco-iridoid pathway from Catharanthus roseus. Nat. Commun. 5, 3606 (2014).

6. Franke, J. et al. Gene discovery in gelsemium highlights conserved gene clusters in monoterpene indole alkaloid biosynthesis. Chembiochem 20, 83-87 (2019).

7. Nakabayashi, R. et al. Metabolomics with (15)N labeling for characterizing missing monoterpene indole alkaloids in plants. Anal. Chem. 92, 5670-5675 (2020).

8. Sharma, A., Amin, D., Sankaranarayanan, A., Arora, R. \& Mathur, A. K. Present status of Catharanthus roseus monoterpenoid indole alkaloids engineering in homo- and hetero-logous systems. Biotechnol. Lett. 42, 11-23 (2020).

9. Pan, Q. F., Mustafa, N. R., Tang, K. X., Choi, Y. H. \& Verpoorte, R. Monoterpenoid indole alkaloids biosynthesis and its regulation in Catharanthus roseus: a literature review from genes to metabolites. Phytochem Rev. 15, 221-250 (2016).

10. Saklani, A. \& Kutty, S. K. Plant-derived compounds in clinical trials. Drug Discov. Today 13, 161-171 (2008).

11. Sriram, D., Yogeeswari, P., Thirumurugan, R. \& Bal, T. R. Camptothecin and its analogues: a review on their chemotherapeutic potential. Nat. Prod. Res. 19 393-412 (2005).

12. Sudo, H., Yamakawa, T., Yamazaki, M., Aimi, N. \& Saito, K. Bioreactor production of camptothecin by hairy root cultures of Ophiorrhiza pumila. Biotechnol. Lett. 24, 359-363 (2002). 
13. Wang, C. et al. Transcription factor $O p W R K Y 3$ is involved in the development and biosynthesis of camptothecin and its precursors in Ophiorrhiza pumila hairy roots. Int. J. Mol. Sci. 20, 3996 (2019).

14. Sirikantaramas, S., Yamazaki, M. \& Saito, K. Mutations in topoisomerase I as a self-resistance mechanism coevolved with the production of the anticancer alkaloid camptothecin in plants. Proc. Natl Acad. Sci. USA 105, 6782-6786 (2008).

15. Yamazaki, M. et al. Coupling deep transcriptome analysis with untargeted metabolic profiling in Ophiorrhiza pumila to further the understanding of the biosynthesis of the anti-cancer alkaloid camptothecin and anthraquinones. Plant Cell Physiol. 54, 686-696 (2013).

16. Shi, M. et al. Targeted metabolic engineering of committed steps improves anti-cancer drug camptothecin production in Ophiorrhiza pumila hairy roots. Ind. Crops Prod. 148, 112277 (2020).

17. Viraporn, V. et al. Correlation of camptothecin-producing ability and phylogenetic relationship in the genus Ophiorrhiza. Planta Med. 77, 759-764 (2011).

18. Seca, A. M. L. \& Pinto, D. Plant secondary metabolites as anticancer agents: successes in clinical trials and therapeutic application. Int. J. Mol. Sci. 19, 263 (2018).

19. Michael, T. P. \& VanBuren, R. Building near-complete plant genomes. Curr. Opin. Plant Biol. 54, 26-33 (2020).

20. Bickhart, D. M. et al. Single-molecule sequencing and chromatin conformation capture enable de novo reference assembly of the domestic goat genome. Nat. Genet. 49, 643-650 (2017)

21. Rai, A., Yamazaki, M. \& Saito, K. A new era in plant functional genomics. Curr. Opin. Syst. Biol. 15, 58-67 (2019).

22. Koren, S., Walenz, B. P., Berlin, K., Miller, J. R., Bergman, N. H. \& Phillippy, A. M. Canu: scalable and accurate long-read assembly via adaptive k-mer weighting and repeat separation. Genome Res. 27, 722-736 (2017).

23. Chin, C. S. et al. Phased diploid genome assembly with single-molecule realtime sequencing. Nat. Methods 13, 1050-1054 (2016).

24. Sabir, J. S. et al. The nuclear genome of Rhazya stricta and the evolution of alkaloid diversity in a medically relevant clade of Apocynaceae. Sci. Rep. 6 , 33782 (2016)

25. Zhao, D. et al. De novo genome assembly of Camptotheca acuminata, a natural source of the anti-cancer compound camptothecin. GigaScience 6, 1-7 (2017).

26. Murat, F., Armero, A., Pont, C., Klopp, C. \& Salse, J. Reconstructing the genome of the most recent common ancestor of flowering plants. Nat. Genet. 49, 490-496 (2017)

27. Jaillon, O. et al. The grapevine genome sequence suggests ancestral hexaploidization in major angiosperm phyla. Nature 449, 463-467 (2007).

28. Pont, C., Wagner, S., Kremer, A., Orlando, L., Plomion, C. \& Salse, J. Paleogenomics: reconstruction of plant evolutionary trajectories from modern and ancient DNA. Genome Biol. 20, 29 (2019).

29. Denoeud, F. et al. The coffee genome provides insight into the convergent evolution of caffeine biosynthesis. Science 345, 1181-1184 (2014).

30. Waterhouse, R. M. et al. BUSCO applications from quality assessments to gene prediction and phylogenomics. Mol. Biol. Evol. 35, 543-548 (2018).

31. Bombarely, A., Rosli, H. G., Vrebalov, J., Moffett, P., Mueller, L. A. \& Martin, G. B. A draft genome sequence of Nicotiana benthamiana to enhance molecular plant-microbe biology research. Mol. Plant Microbe Interact. 25, 1523-1530 (2012).

32. Tomato Genome, $\mathrm{C}$. The tomato genome sequence provides insights into fleshy fruit evolution. Nature 485, 635-641 (2012).

33. Emms, D. M. \& Kelly, S. OrthoFinder: phylogenetic orthology inference for comparative genomics. Genome Biol. 20, 238 (2019).

34. Yang, Z. PAML 4: phylogenetic analysis by maximum likelihood. Mol. Biol. Evol. 24, 1586-1591 (2007).

35. Jiao, Y. et al. A genome triplication associated with early diversification of the core eudicots. Genome Biol. 13, R3 (2012).

36. Panchy, N., Lehti-Shiu, M. \& Shiu, S. H. Evolution of gene duplication in plants. Plant Physiol. 171, 2294-2316 (2016).

37. Contreras, B., Vives, C., Castells, R. \& Casacuberta, J. M. In Evolutionary Biology: Biodiversification from Genotype to Phenotype (ed. Pontarotti, P.) (Springer International Publishing, 2015)

38. Vicient, C. M. \& Casacuberta, J. M. Impact of transposable elements on polyploid plant genomes. Ann. Bot. 120, 195-207 (2017).

39. Lichman, B. R., Godden, G. T. \& Buell, C. R. Gene and genome duplications in the evolution of chemodiversity: perspectives from studies of Lamiaceae. Curr. Opin. Plant Biol. 55, 74-83 (2020).

40. Tsugawa, H. et al. A cheminformatics approach to characterize metabolomes in stable-isotope-labeled organisms. Nat. Methods 16, 295-298 (2019).

41. Nakabayashi, R. \& Saito, K. Higher dimensional metabolomics using stable isotope labeling for identifying the missing specialized metabolism in plants. Curr. Opin. Plant Biol. 55, 84-92 (2020).
42. Sadre, R. et al. Metabolite diversity in alkaloid biosynthesis: a multilane (diastereomer) highway for camptothecin synthesis in Camptotheca acuminata. Plant Cell 28, 1926-1944 (2016).

43. Yang, Y. et al. Bifunctional cytochrome P450 enzymes involved in camptothecin biosynthesis. ACS Chem. Biol. 14, 1091-1096 (2019)

44. Lynch, M. \& Conery, J. S. The evolutionary fate and consequences of duplicate genes. Science 290, 1151-1155 (2000)

45. Carretero-Paulet, L. \& Fares, M. A. Evolutionary dynamics and functional specialization of plant paralogs formed by whole and small-scale genome duplications. Mol. Biol. Evol. 29, 3541-3551 (2012).

46. Copley, S. D. Evolution of new enzymes by gene duplication and divergence. FEBS J. 287, 1262-1283 (2020).

47. Moore, R. C. \& Purugganan, M. D. The evolutionary dynamics of plant duplicate genes. Curr. Opin. Plant Biol. 8, 122-128 (2005).

48. Murrell, B. et al. Gene-wide identification of episodic selection. Mol. Biol. Evol. 32, 1365-1371 (2015).

49. Wisecaver, J. H., Borowsky, A. T., Tzin, V., Jander, G., Kliebenstein, D. J. \& Rokas, A. A global coexpression network approach for connecting genes to specialized metabolic pathways in plants. Plant Cell 29, 944-959 (2017).

50. Kautsar, S. A., Duran, H. G. S., Blin, K., Osbourn, A. \& Medema, M. H. plantiSMASH: automated identification, annotation and expression analysis of plant biosynthetic gene clusters. Nucleic Acids Res. 45, W55-W63 (2017).

51. De Luca, V. \& St Pierre, B. The cell and developmental biology of alkaloid biosynthesis. Trends Plant Sci. 5, 168-173 (2000).

52. Shimizu, Y. et al. Metabolic diversification of nitrogen-containing metabolites by the expression of a heterologous lysine decarboxylase gene in Arabidopsis. Plant J. 100, 505-521 (2019).

53. Wink, M. Evolution of secondary metabolites from an ecological and molecular phylogenetic perspective. Phytochemistry 64, 3-19 (2003).

54. Souard, F. et al. Metabolomics fingerprint of coffee species determined by untargeted-profiling study using LC-HRMS. Food Chem. 245, 603-612 (2018).

55. Nutzmann, H. W., Huang, A. \& Osbourn, A. Plant metabolic clusters - from genetics to genomics. New Phytol. 211, 771-789 (2016).

56. $\mathrm{Li}, \mathrm{Q}$. et al. Gene clustering and copy number variation in alkaloid metabolic pathways of opium poppy. Nat. Commun. 11, 1190 (2020).

57. Nutzmann, H. W. et al. Active and repressed biosynthetic gene clusters have spatially distinct chromosome states. Proc. Natl Acad. Sci. USA 117, 13800-13809 (2020)

58. Itkin, M. et al. Biosynthesis of antinutritional alkaloids in solanaceous crops is mediated by clustered genes. Science 341, 175-179 (2013).

59. Yeaman, S. Genomic rearrangements and the evolution of clusters of locally adaptive loci. Proc. Natl Acad. Sci. USA 110, E1743-E1751 (2013).

60. Jiao, W. B. \& Schneeberger, K. The impact of third generation genomic technologies on plant genome assembly. Curr. Opin. Plant Biol. 36, 64-70 (2017).

61. Ghurye, J. \& Pop, M. Modern technologies and algorithms for scaffolding assembled genomes. PLoS Comput. Biol. 15, e1006994 (2019).

62. Kitajima, M., Nakamura, M., Takayama, H., Saito, K., Stockigt, J. \& Aimi, N. Constituents of regenerated plants of Ophiorrhiza pumila; formation of a new glycocamptothecin and predominant formation of (3R)-deoxypumiloside over (3S)-congener. Tetrahedron Lett. 38, 8997-9000 (1997).

63. Asano, T. et al. Suppression of camptothecin biosynthetic genes results in metabolic modification of secondary products in hairy roots of Ophiorrhiza pumila. Phytochemistry 91, 128-139 (2013).

64. Kitajima, M. et al. Camptothecin-related alkaloids from hairy roots of Ophiorrhiza pumila. Tetrahedron 58, 9169-9178 (2002).

65. Rohani, E. R. et al. An MYB transcription factor regulating specialized metabolisms in Ophiorrhiza pumila. Plant Biotechnol. 33, 1-+ (2016).

66. Lou, Q. F. et al. Single-copy gene-based chromosome painting in cucumber and its application for chromosome rearrangement analysis in Cucumis. Plant J. 78, 169-179 (2014)

67. Chikhi, R. \& Medvedev, P. Informed and automated k-mer size selection for genome assembly. Bioinformatics 30, 31-37 (2014).

68. Pfosser, M., Amon, A., Lelley, T. \& Heberlebors, E. Evaluation of sensitivity of flow-cytometry in detecting aneuploidy in wheat using disomic and ditelosomic wheat-rye addition lines. Cytometry 21, 387-393 (1995).

69. Kronenberg, Z. N. et al. Extended haplotype phasing of de novo genome assemblies with FALCON-Phase. Preprint at https://doi.org/10.1101/327064 (2019).

70. Li, H. \& Durbin, R. Fast and accurate short read alignment with BurrowsWheeler transform. Bioinformatics 25, 1754-1760 (2009).

71. Jibran, R. et al. Chromosome-scale scaffolding of the black raspberry (Rubus occidentalis $\mathrm{L}$.) genome based on chromatin interaction data. Horticulture Res. 5, 8 (2018).

72. Burton, J. N., Adey, A., Patwardhan, R. P., Qiu, R., Kitzman, J. O. \& Shendure, J. Chromosome-scale scaffolding of de novo genome assemblies based on chromatin interactions. Nat. Biotechnol. 31, 1119-1125 (2013). 
73. English, A. C. et al. Mind the gap: upgrading genomes with Pacific Biosciences RS long-read sequencing technology. PloS ONE 7, e47768 (2012).

74. Walker, B. J. et al. Pilon: an integrated tool for comprehensive microbial variant detection and genome assembly improvement. PloS ONE 9, e112963 (2014).

75. Bolger, A. M., Lohse, M. \& Usadel, B. Trimmomatic: a flexible trimmer for Illumina sequence data. Bioinformatics 30, 2114-2120 (2014).

76. Langmead, B. \& Salzberg, S. L. Fast gapped-read alignment with Bowtie 2. Nat. Methods 9, 357-359 (2012)

77. Durand, N. C. et al. Juicer provides a one-click system for analyzing loopresolution Hi-C experiments. Cell Syst. 3, 95-98 (2016).

78. Yuan, Y., Bayer, P. E., Scheben, A., Chan, C. K. \& Edwards, D. BioNanoAnalyst: a visualisation tool to assess genome assembly quality using BioNano data. BMC Bioinformatics 18, 323 (2017).

79. Combes, M. C. et al. Characterization of microsatellite loci in Coffea arabica and related coffee species. Mol. Ecol. 9, 1178-1180 (2000).

80. Tran, H. T. M., Ramaraj, T., Furtado, A., Lee, L. S. \& Henry, R. J. Use of a draft genome of coffee (Coffea arabica) to identify SNPs associated with caffeine content. Plant Biotechnol. J. 16, 1756-1766 (2018).

81. Grabherr, M. G. et al. Full-length transcriptome assembly from RNA-Seq data without a reference genome. Nat. Biotechnol. 29, 644-652 (2011)

82. Kent, W. J. BLAT-the BLAST-like alignment tool. Genome Res. 12, 656-664 (2002).

83. Hirakawa, H. et al. De novo whole-genome assembly in Chrysanthemum seticuspe, a model species of Chrysanthemums, and its application to genetic and gene discovery analysis. DNA Res. 26, 195-203 (2019).

84. Hoff, K. J., Lomsadze, A., Borodovsky, M. \& Stanke, M. Whole-genome annotation with BRAKER. Methods Mol. Biol. 65-95, 2019 (1962).

85. Edwards, K. D. et al. A reference genome for Nicotiana tabacum enables mapbased cloning of homeologous loci implicated in nitrogen utilization efficiency. BMC genomics 18, 448 (2017).

86. De Vega, J. J. et al. Red clover (Trifolium pratense L.) draft genome provides a platform for trait improvement. Sci. Rep. 5, 17394 (2015)

87. Campbell, M. S. et al. MAKER-P: a tool kit for the rapid creation, management, and quality control of plant genome annotations. Plant Physiol. 164, 513-524 (2014).

88. Stanke, M., Diekhans, M., Baertsch, R. \& Haussler, D. Using native and syntenically mapped cDNA alignments to improve de novo gene finding. Bioinformatics 24, 637-644 (2008).

89. Korf, I. Gene finding in novel genomes. BMC Bioinformatics 5, 59 (2004).

90. Lomsadze, A., Ter-Hovhannisyan, V., Chernoff, Y. O. \& Borodovsky, M. Gene identification in novel eukaryotic genomes by self-training algorithm. Nucleic Acids Res. 33, 6494-6506 (2005).

91. Trapnell, C. et al. Differential gene and transcript expression analysis of RNAseq experiments with TopHat and Cufflinks. Nat. Protoc. 7, 562-578 (2012).

92. Jones, P. et al. InterProScan 5: genome-scale protein function classification. Bioinformatics 30, 1236-1240 (2014).

93. Llorens, C. et al. The Gypsy Database (GyDB) of mobile genetic elements: release 2.0. Nucleic Acids Res. 39, D70-D74 (2011).

94. Jin, J. et al. PlantTFDB 4.0: toward a central hub for transcription factors and regulatory interactions in plants. Nucleic Acids Res. 45, D1040-D1045 (2017).

95. Chan, P. P. \& Lowe, T. M. tRNAscan-SE: searching for tRNA genes in genomic sequences. Methods Mol. Biol. 1962, 1-14 (2019).

96. Nawrocki, E. P. \& Eddy, S. R. Infernal 1.1: 100-fold faster RNA homology searches. Bioinformatics 29, 2933-2935 (2013).

97. Kalvari, I. et al. Rfam 13.0: shifting to a genome-centric resource for noncoding RNA families. Nucleic Acids Res. 46, D335-D342 (2018).

98. Bao, W., Kojima, K. K. \& Kohany, O. Repbase Update, a database of repetitive elements in eukaryotic genomes. Mobile DNA 6, 11 (2015).

99. Xu, Z. \& Wang, H. LTR_FINDER: an efficient tool for the prediction of fulllength LTR retrotransposons. Nucleic Acids Res. 35, W265-W268 (2007).

100. Price, A. L., Jones, N. C. \& Pevzner, P. A. De novo identification of repeat families in large genomes. Bioinformatics 21, i351-i358 (2005).

101. Tarailo-Graovac, M. \& Chen, N. Using RepeatMasker to identify repetitive elements in genomic sequences. Curr. Protoc. Bioinformatics Chapter 4, Unit 4.10 (2009).

102. Benson, G. Tandem repeats finder: a program to analyze DNA sequences. Nucleic Acids Res. 27, 573-580 (1999).

103. Kielbasa, S. M., Wan, R., Sato, K., Horton, P. \& Frith, M. C. Adaptive seeds tame genomic sequence comparison. Genome Res. 21, 487-493 (2011).

104. Wang, Y. et al. MCScanX: a toolkit for detection and evolutionary analysis of gene synteny and collinearity. Nucleic Acids Res. 40, e49 (2012).

105. Amborella Genome, P. The Amborella genome and the evolution of flowering plants. Science 342, 1241089 (2013).

106. Lamesch, P. et al. The Arabidopsis Information Resource (TAIR): improved gene annotation and new tools. Nucleic Acids Res. 40, D1202-D1210 (2012).

107. Badouin, $\mathrm{H}$. et al. The sunflower genome provides insights into oil metabolism, flowering and Asterid evolution. Nature 546, 148-152 (2017).
108. Hane, J. K. et al. A comprehensive draft genome sequence for lupin (Lupinus angustifolius), an emerging health food: insights into plant-microbe interactions and legume evolution. Plant Biotechnol. J. 15, 318-330 (2017)

109. Guo, L. et al. The opium poppy genome and morphinan production. Science 362, 343-347 (2018).

110. Enright, A. J., Van Dongen, S. \& Ouzounis, C. A. An efficient algorithm for large-scale detection of protein families. Nucleic Acids Res. 30, 1575-1584 (2002).

111. Edgar, R. C. MUSCLE: multiple sequence alignment with high accuracy and high throughput. Nucleic Acids Res. 32, 1792-1797 (2004).

112. Filiault, D. L. et al. The Aquilegia genome provides insight into adaptive radiation and reveals an extraordinarily polymorphic chromosome with a unique history. Elife 7, e36426 (2018).

113. Zhang, L. et al. Improved Brassica rapa reference genome by single-molecule sequencing and chromosome conformation capture technologies. Hortic. Res. 5, 50 (2018)

114. Varshney, R. K. et al. Draft genome sequence of chickpea (Cicer arietinum) provides a resource for trait improvement. Nat. Biotechnol. 31, 240-246 (2013).

115. $\mathrm{Wu}, \mathrm{G}$. A. et al. Sequencing of diverse mandarin, pummelo and orange genomes reveals complex history of admixture during citrus domestication. Nat. Biotechnol. 32, 656-662 (2014).

116. Woycicki, R. et al. The genome sequence of the North-European cucumber (Cucumis sativus L.) unravels evolutionary adaptation mechanisms in plants. PloS ONE 6, e22728 (2011).

117. Schmutz, J. et al. Genome sequence of the palaeopolyploid soybean. Nature 463, 178-183 (2010).

118. Mochida, K. et al. Draft genome assembly and annotation of Glycyrrhiza uralensis, a medicinal legume. Plant J. 89, 181-194 (2017).

119. Paterson, A. H. et al. Repeated polyploidization of Gossypium genomes and the evolution of spinnable cotton fibres. Nature 492, 423-427 (2012).

120. Velasco, R. et al. The genome of the domesticated apple (Malus $\mathrm{x}$ domestica Borkh.). Nat. Genet. 42, 833-839 (2010).

121. Young, N. D. et al. The Medicago genome provides insight into the evolution of rhizobial symbioses. Nature 480, 520-524 (2011).

122. Droc, G. et al. The banana genome hub. Database 2013, bat035 (2013).

123. Ming, R. et al. Genome of the long-living sacred lotus (Nelumbo nucifera Gaertn.). Genome Biol. 14, R41 (2013).

124. Ouyang, S. et al. The TIGR Rice Genome Annotation Resource: improvements and new features. Nucleic Acids Res. 35, D883-D887 (2007).

125. Tuskan, G. A. et al. The genome of black cottonwood, Populus trichocarpa (Torr. \& Gray). Science 313, 1596-1604 (2006).

126. International Peach Genome, I. et al. The high-quality draft genome of peach (Prunus persica) identifies unique patterns of genetic diversity, domestication and genome evolution. Nat. Genet. 45, 487-494 (2013).

127. Banks, J. A. et al. The Selaginella genome identifies genetic changes associated with the evolution of vascular plants. Science 332, 960-963 (2011).

128. Motamayor, J. C. et al. The genome sequence of the most widely cultivated cacao type and its use to identify candidate genes regulating pod color. Genome Biol. 14, r53 (2013).

129. Hirsch, C. N. et al. Draft assembly of elite inbred line PH207 provides insights into genomic and transcriptome diversity in maize. Plant Cell 28, 2700-2714 (2016).

130. Stamatakis, A. RAxML version 8: a tool for phylogenetic analysis and postanalysis of large phylogenies. Bioinformatics 30, 1312-1313 (2014).

131. Puttick, M. N. MCMCtreeR: functions to prepare MCMCtree analyses and visualize posterior ages on trees. Bioinformatics 35, 5321-5322 (2019).

132. Kumar, S., Stecher, G., Suleski, M. \& Hedges, S. B. TimeTree: a resource for timelines, timetrees, and divergence times. Mol. Biol. Evol. 34, 1812-1819 (2017).

133. Fu, L., Niu, B., Zhu, Z., Wu, S. \& Li, W. CD-HIT: accelerated for clustering the next-generation sequencing data. Bioinformatics 28, 3150-3152 (2012).

134. Csuros, M. Count: evolutionary analysis of phylogenetic profiles with parsimony and likelihood. Bioinformatics 26, 1910-1912 (2010).

135. Tsugawa, $H$. et al. Hydrogen rearrangement rules: computational MS/MS fragmentation and structure elucidation using MS-FINDER software. Anal. Chem. 88, 7946-7958 (2016).

136. Rai, A. et al. Multiomics-based characterization of specialized metabolites biosynthesis in Cornus officinalis. DNA Res. 27, dsaa009 (2020).

137. Haas, B. J. et al. De novo transcript sequence reconstruction from RNA-seq using the Trinity platform for reference generation and analysis. Nat. Protoc. 8, 1494-1512 (2013).

138. Anders, S., Pyl, P. T. \& Huber, W. HTSeq-a Python framework to work with high-throughput sequencing data. Bioinformatics 31, 166-169 (2015).

139. Villanueva, R. A. M. \& Chen, Z. J. In Measurement: Interdisciplinary Research and Perspectives 2nd ed, Vol. 17, 160-167 (2019).

140. Schlapfer, P. et al. Genome-wide prediction of metabolic enzymes, pathways, and gene clusters in plants. Plant Physiol. 173, 2041-2059 (2017). 
141. Chae, L., Kim, T., Nilo-Poyanco, R. \& Rhee, S. Y. Genomic signatures of specialized metabolism in plants. Science 344, 510-513 (2014).

142. Caspi, R. et al. The MetaCyc database of metabolic pathways and enzymes. Nucleic Acids Res. 46, D633-D639 (2018).

143. Karp, P. D., Latendresse, M. \& Caspi, R. The pathway tools pathway prediction algorithm. Stand. Genom. Sci. 5, 424-429 (2011).

144. Nakamura, T., Yamada, K. D., Tomii, K. \& Katoh, K. Parallelization of MAFFT for large-scale multiple sequence alignments. Bioinformatics 34, 2490-2492 (2018).

145. Huerta-Cepas, J., Serra, F. \& Bork, P. ETE 3: reconstruction, analysis, and visualization of phylogenomic data. Mol. Biol. Evol. 33, 1635-1638 (2016).

146. Rai, A. et al. Chromosome-level genome assembly of Ophiorrhiza pumila reveals the evolution of camptothecin biosynthesis. Zenodo https://doi.org/ 10.5281/zenodo.4279079 (2020).

\section{Acknowledgements}

The super-computing resource was provided by the National Institute of Genetics, Research Organization of Information and Systems, Japan. The computing resources were provided by the National Institute of Genetics, Research Organization of Information and Systems, and the Medical Mycology Research Center, Chiba University, Japan. We thank Michimi Nakamura (Chiba University) and Kenji Uemukai (AS ONE) to assist in the high-molecular-weight genomic DNA extraction for PacBio and Bionano optical map sequencing, respectively. We also thank our colleague, Dr. Gourvendu Saxena from the National University of Singapore, Singapore, and Dr. Sheelendra Pratap Singh from CSIR-Indian Institute of Toxicology Research, India, for helping us to improve the content of the manuscript. This study was supported by Grant-in-Aid for Scientific Research on Innovative Areas, MEXT (16H06454 to M.Y.); Grant-in-Aid for Early-Career Scientists, JSPS (19K16391 to A.R.); Grant-in-Aid for Scientific Research (S), JSPS (19H05652 to K.S.); a research and development grant of the Japan Agency for Medical Research and Development (AMED; 17ak0101046h0002 to M.Y.); Kobayashi Foundation (to K.S. and M.Y.); JSPS KAKENHI (18H02432, 18 K19155 to H.T.), Sequencing support from PAGS, JSPS (16H06279; PAGS); Chiba University GP Program (to K.S.), and Kazusa DNA Research Institute Foundation.

\section{Author contributions}

A.R., K.S., and M.Y. conceptualize the project. A.R. and T.N. prepared all material for sequencing, isotope labeling, and metabolite profiling. A.T. performed genome sequencing. R.N. and T.M. performed metabolite profiling. R.N., A.R., and H.T. analyzed and annotated metabolome datasets. R.F. and S.K. performed FISH experiment. S.K., S.H., and E.G. prepared and provided plant material for FISH analysis. S.K. provided FISH results interpretation. A.R., K.H., and M.R. performed transcriptome analysis and gene cluster analysis. A.R. and H.H. performed genome assembly. A.R., H.H., and H.N. worked to derive phased genome assembly. H.H. predicted gene models and annotation H.U. performed flow cytometer-based genome size estimation. A.R. and Y.K. prepared Hi-C library for sequencing. A.R. performed comparative genome analysis. A.R., H.H., H.T., R.N., K.S., and M.Y. contributed toward interpretation of genome and metabolome analysis results. A.R., H.H., M.R., and K.H. prepared all figures and tables. A.R., K.S., and M.Y. wrote the paper.

\section{Competing interests}

The authors declare no competing interests.

\section{Additional information}

Supplementary information is available for this paper at https://doi.org/10.1038/s41467020-20508-2.

Correspondence and requests for materials should be addressed to A.R. or M.Y.

Peer review information Nature Communications thanks Elisabeth Kaltenegger, Benoit St Pierre, Michael Wink and the other, anonymous, reviewer(s) for their contribution to the peer review of this work. Peer reviewer reports are available.

Reprints and permission information is available at http://www.nature.com/reprints

Publisher's note Springer Nature remains neutral with regard to jurisdictional claims in published maps and institutional affiliations.

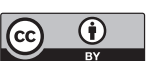

Open Access This article is licensed under a Creative Commons Attribution 4.0 International License, which permits use, sharing, adaptation, distribution and reproduction in any medium or format, as long as you give appropriate credit to the original author(s) and the source, provide a link to the Creative Commons license, and indicate if changes were made. The images or other third party material in this article are included in the article's Creative Commons license, unless indicated otherwise in a credit line to the material. If material is not included in the article's Creative Commons license and your intended use is not permitted by statutory regulation or exceeds the permitted use, you will need to obtain permission directly from the copyright holder. To view a copy of this license, visit http://creativecommons.org/ licenses/by/4.0/.

(c) The Author(s) 2021 